This item was submitted to Loughborough's Research Repository by the author.

Items in Figshare are protected by copyright, with all rights reserved, unless otherwise indicated.

\title{
Measurement of the spatially distributed temperature and soot loadings in a laminar diffusion flame using a Cone-Beam Tomography technique
}

PLEASE CITE THE PUBLISHED VERSION

http://dx.doi.org/10.1016/j.jqsit.2013.07.024

\section{PUBLISHER}

(C) Elsevier

VERSION

AM (Accepted Manuscript)

\section{PUBLISHER STATEMENT}

This work is made available according to the conditions of the Creative Commons Attribution-NonCommercialNoDerivatives 4.0 International (CC BY-NC-ND 4.0) licence. Full details of this licence are available at: https://creativecommons.org/licenses/by-nc-nd/4.0/

\section{LICENCE}

CC BY-NC-ND 4.0

\section{REPOSITORY RECORD}

Zhao, Huayong, Ben Williams, and Richard Stone. 2019. "Measurement of the Spatially Distributed Temperature and Soot Loadings in a Laminar Diffusion Flame Using a Cone-beam Tomography Technique". figshare. https://hdl.handle.net/2134/20060. 


\title{
Measurement of the Spatially Distributed Temperature and Soot Loadings in a Laminar Diffusion Flame Using a Cone-Beam Tomography Technique
}

\author{
Huayong Zhao ${ }^{\mathrm{a}^{*}}$, Ben Williams ${ }^{\mathrm{b}}$, Richard Stone ${ }^{\mathrm{a}}$ \\ ${ }^{a}$ Department of Engineering Science, University of Oxford, Parks Road, Oxford, U.K., OX1 3PJ \\ ${ }^{b}$ Department of Physics, University of Oxford, Parks Road, Oxford, U.K., OX1 3PU
}

\begin{abstract}
A new low-cost optical diagnostic technique, called Cone Beam Tomographic Three Colour Spectrometry (CBT-TCS), has been developed to measure the planar distributions of temperature, soot particle size, and soot volume fraction in a co-flow axi-symmetric laminar diffusion flame. The image of a flame is recorded by a colour camera, and then by using colour interpolation and applying a cone beam tomography algorithm, a colour map can be reconstructed that corresponds to a diametral plane. Look-up tables calculated using Planck's law and different scattering models are then employed to deduce the temperature, approximate average soot particle size and soot volume fraction in each voxel (volumetric pixel). A sensitivity analysis of the look-up tables shows that the results have a high temperature resolution but a relatively low soot particle size resolution. The assumptions underlying the technique are discussed in detail. Sample data from an ethylene laminar diffusion flame are compared with data in the literature for similar flames. The comparison shows very consistent temperature and soot volume fraction profiles. Further analysis indicates that the difference seen in comparison with published results are within the measurement uncertainties. This methodology is ready to be applied to measure 3D data by capturing multiple flame images from different angles for non-axisymmetric flame.
\end{abstract}

Keywords: temperature; soot diameter; soot volume fraction; tomography; laminar diffusion flame

\section{Introduction}

As more and more concerns have been raised about the negative impact of particulate emissions from the combustion of hydrocarbons to human health and the environment, much research has been carried out to investigate the formation mechanisms of the particulates and possible abatement methods. This research is based on the measurement of the combustion environment, especially temperature, and the corresponding soot loadings, including their size and concentration. Amongst different measurement techniques, optical diagnostics have many advantages, including their non-intrusive characteristics and good spatial and temporal resolution.

Non-laser based optical diagnostics of soot can be broadly divided into two groups: emission based measurements and extinction based measurements. The former do not need a light source so are less complicated in their experimental setup and are easy to apply. The emission based measurement techniques are usually based on thermal radiation from the soot particles. The emissivity of such particles can be calculated from a scattering model. Typical scattering models using the assumption of spherical soot particles include the Hottel and Broughton empirical correlation [1] (used in two-colour or three-colour pyrometry), the RayleighGans (RG) theory (used in most single or multiple wavelength soot spectrometry applications) [2], and the Mie scattering theory, which is the analytical solution of Maxwell's equations for scattering by spherical particles [3].

Meanwhile, tomography, which is used to reconstruct a higher dimensional property field based on projections in a lower dimension, has been applied to line-of-sight measurements to obtain spatial resolution along the optical path. Hall and Boncyzk [4] used single wavelength emission-absorption spectrometry to reconstruct the 2D soot concentration and temperature fields in ethylene and iso-octane diffusion flames at specific heights. Snelling et al. [5] extended this work by applying multi-wavelength emission spectrometry to measure the 2D temperature and soot concentration fields and they also discussed the influence of various optical parameters on the accuracy of the experiment. Ayranci et al. [6] proposed a new approach to correct the assumption of a constant refractive index for soot over a certain range of wavelengths when using the RG theory. Lu et al. [7] introduced an iterative method to diminish the error caused by the optically-thin assumption. These researchers have greatly improved the spatial resolution and accuracy of two-dimensional emission spectrometry. Their experiments are all based on the measurement of absolute light intensity, which can be difficult because of the need for a source with an absolute calibration, such as a black body at a known temperature. These experiments 
also rely on the assumption that the RG theory is valid throughout the whole spectrum, which is not necessarily the case, especially in the visible light region. In addition, two-dimensional tomographic emission spectrometry only gives the temperatures and soot concentration distributions at a specific height in one measurement. Moreover, their measurements do not include any information about the soot particle size.

The technique introduced in this paper, called Cone Beam Tomographic Three Colour Spectrometry (CBTTCS), is a relatively inexpensive analysis method based on a modified three colour pyrometry approach combined with a 3D cone beam tomography technique. CBT-TCS can be used to measure the temperature and soot diameter distributions without needing an absolute calibration, but the measurement of soot volume fraction does need an absolute intensity calibration. In general this will require multiple views, but in the case of an axisymmetric flame only one image is needed.

This paper will begin by reviewing selected thermal radiation and scattering theory along with introducing how this is relevant to the CBT-TCS technique. The sensitivity of the technique to changes in temperature and particle size is then assessed numerically. The paper continues by explaining how CBT-TCS is realised, both in terms of apparatus and going into some detail regarding the computer algorithm. Following this is a discussion of any further assumptions related to the technique. Finally, this paper shows results from applying CBT-TCS to a laminar diffusion flame (the Gülder burner [8]), before closing with some conclusions that discuss the strengths and weaknesses of this measurement approach.

\section{Theory}

The physics of thermal radiation and light scattering by particles are introduced in this section and used as background in the selection of a scattering model to use in CBT-TCS.

\subsection{Thermal radiation}

The thermal radiation at specific wavelength $\left(I_{\lambda}\right)$ from a surface as a function of temperature $(T)$ can be calculated from Planck's law, by employing a dimensionless material parameter called emissivity $\left(\varepsilon_{\lambda}\right)$, which is simply the ratio of the emitted radiation to that of a perfect black body source.

Assuming a soot particle is in local thermal equilibrium, Kirchhoff's Law explains that the absorption of the particle must balance its emission, for all wavelengths. In the case that the soot particles' refractive index is known, scattering theory may be used to determine the interaction of radiation with the particle. Scattering theory provides values for the efficiency with which radiation is absorbed, $Q_{a b s}$, and scattered, $Q_{\text {scatt }}$. These contributions are also often summed to form the overall extinction efficiency, $Q_{\text {ext }}$. In order for thermal balance to hold, the emissivity $\varepsilon_{\lambda}$ of a soot particle can therefore be determined by calculating $Q_{a b s}$ since the scattering does not involve any energy transfer [9]. $Q_{a b s}$ is generally a function of wavelength, $\lambda$, and can be calculated by using different scattering models, which will be discussed in section 2.2.

Assuming a mono-disperse distribution of independent particles, the spectral radiance of the spherical soot particles $\left(I_{\lambda}^{\prime}\right.$, measured in $\left.\mathrm{Js}^{-1} \mathrm{sr}^{-1} \mathrm{~m}^{-1}\right)$ of diameter $(d)$ within a voxel (volumetric pixel) with volume $V$ can be calculated by adopting the following equation:

$$
I_{\lambda}^{\prime}=\frac{6 f_{v} V}{d} \cdot Q_{a b s} \cdot I_{\lambda}(T)
$$

where $f_{v}$ is the soot volume fraction. This mono-disperse assumption (that the soot particles within an individual voxel have the same shape, size and mass) will introduce some errors into the measurements produced by CBTTCS, depending on the homogeneity of the thermal radiation properties of the soot particles. However, since the voxel size is small (a cube with $46 \mu \mathrm{m}$ side length), the technique may still track ensemble-averaged properties with good spatial resolution.

\subsection{Scattering models}

This sub-section discusses scattering models and particle characteristics.

\subsubsection{Spherical particles}

A solution of Maxwell's equations for scattering from spherical particles was first derived by Mie in 1908 [3]. The mathematical description of absorption efficiency as predicted by Mie theory can be written as: 


$$
Q_{a b s}^{M}=\frac{2}{x^{2}} \sum_{n=1}^{\infty}(2 n+1)\left(\operatorname{Re}\left(a_{n}+b_{n}\right)-\left(\left|a_{n}\right|^{2}+\left|b_{n}\right|^{2}\right)\right)
$$

where $x$ is the size parameter which is defined as $x=\pi d / \lambda$ ( $d$ is the diameter of the particle) and $a_{n}$ and $b_{n}$ are scattering coefficients which are functions of the complex refractive index $(m=n-\mathrm{i} k)$ and the size parameter. Further details such as expansions of $a_{n}$ and $b_{n}$ can be found in ref. [10]. When particle size is small compared to the incident light wavelength, Mie theory can be simplified to the Rayleigh-Gans (RG) theory, which is widely applied because of its low computational cost. In this case, the absorption efficiency can be calculated by:

$$
Q_{a b s}^{R}=4 x \operatorname{Im}\left\{\frac{m^{2}-1}{m^{2}+2}\right\}=4 x E(m)
$$

where $E(m)$ is usually referred to as the soot absorption function. As the size parameter becomes larger, the RG theory becomes less accurate and it can be improved by a fitting correlation introduced by Penndorf [11]:

$$
\begin{aligned}
& \qquad Q_{a b s}^{P}=Q_{a b s}^{R}+2 x^{2}\left[N_{1}\left(\frac{1}{15}+\frac{5}{3} \frac{1}{M_{4}}+\frac{6}{5} \frac{M_{5}}{M_{1}^{2}}\right)+\frac{4}{3} \frac{M_{6}}{M_{1}^{2}} x\right]-Q_{s c a}^{R}\left[1+2 \frac{x^{2}}{M 1}\left(\frac{3}{5} M_{3}-2 N_{1} x\right)\right] \quad \text { eq. (4) } \\
& \text { where } N_{1}=2 n k, N_{2}=n^{2}-k^{2}, N_{3}=N_{1}^{2}+N_{2}^{2}, M_{1}=N_{1}^{2}+\left(2+N_{2}\right)^{2}, M_{2}=1+2 N_{2}, M_{3}=N_{3}-4, \\
& \qquad M_{4}=4 N_{1}^{2}+\left(3+2 N_{2}\right)^{2}, M_{5}=4\left(N_{2}-5\right)+7 N_{3}, M_{6}=\left(N_{2}+N_{3}-2\right)^{2}-9 N_{1}^{2}
\end{aligned}
$$

Fig. 1 Comparison of scattering models (RG in the legend stands for Rayleigh-Gans theory). Top left: the variation of absorption efficiency as a function of size parameter $(x=\pi d / \lambda)$. Bottom left: deviations from Mie theory predictions. Top right: the relative strength of scattering to absorption, which shows that scattering becomes more important as the size parameter increases. Bottom right: particle size parameter versus particle diameter and incident wavelength, used to understand the other three plots in terms of physical parameters.

The accuracy of the RG theory and the Penndorf extension can be seen from fig. 1 where the complex refractive index is assumed constant $(m=1.55-0.55 \mathrm{i})$ within the visible spectrum. The error with respect to the Mie theory is defined as:

$$
\text { error }=\left|\frac{Q^{R \text { or } P}-Q^{M}}{Q^{M}}\right| * 100 \%
$$

The rightmost subplot on the top row of fig. 1 indicates that when using the light extinction method to measure the temperature and soot loading, then scattering must be taken into account for accurate results when the size parameter is larger than 0.6. The bottom left error plot in fig. 1 shows that to achieve less than $10 \%$ error with respect to the Mie theory, the maximum size parameter when using the RG theory is around 0.45 , increasing to 0.8 when using the Penndorf extension. According to the contour plot of the size parameter in the bottom right of fig. 1, the corresponding maximum particle diameter ranges for using RG theory and the Penndorf extension in the visible spectrum are 58-114 $\mathrm{nm}$ and 101-204 $\mathrm{nm}$ respectively.

\subsubsection{The complex refractive index of soot particles}

Whether a constant $E(m)$ can be used for visible spectrum is highly debated. Coderre et al. [12] suggested that a constant $E(m)$ can be used in the visible spectrum for soot particles after they investigated the optical properties of aged and cooled soot particles in methane flames with detailed information about the soot morphology. They also reviewed several scattering models used to calculate the spectrally-dependant $E(m)$ and suggested that those models should be used with care because they assumed the same optical properties between soot particles and carbon, and this has yet to be verified by experiments. Migliorini et al. [13] investigated the optical properties of soot by using the line-of-sight-attenuation (LOSA) method in different regions of the flame and they reported that $E(m)$ is sensitive to both wavelength and location in the flame for wavelengths less than $700 \mathrm{~nm}$. They attribute the difference in light attenuation to the absorbance of large PAH (Polycyclic Aromatic Hydrocarbon) molecules, condensed species and nanoparticles. The data they reported show that the variation in $E(m)$ is below $10 \%$ across the visible spectrum and in regions of the flame higher than $10 \mathrm{~mm}$ above the exit of the fuel tube. Fig. 2 shows the effect of refractive index on absorption efficiency $\left(Q_{a b s}\right)$ when using the Mie theory. This figure show that $Q_{a b s}$ is insensitive to the real part of the refractive index $(n)$ as expected because $n$ decides the phase speed while it is sensitive to the imaginary part $(k) .10 \%$ difference in refractive index typically results in around $10 \%$ difference in absorption efficiency.

Fig. 2 Effect of refractive index on absorption efficiency when using the Mie theory 
In the absence of a firm conclusion regarding the refractive index of soot under these experimental conditions, the analysis presented in this paper uses a constant $m$ value $(1.55-0.55 i)$. This value is the average of those reported in the literature [14].

\subsubsection{Non-spherical particles and particle aggregates}

Many articles have reported that the soot particles in hydrocarbon flames are fractal aggregates (i.e. complex, branched, chain-like structures) consisting of different sizes of spherical primary particles [12, 13, 15]. A review on the scattering properties of soot aggregates has been made by Sorensen [15]. Based on the assumptions of spherical primary particles and point contacts between primary particles, several exact scattering theories have been investigated by different researchers, such as the Generalized Mie Method (GMM) [16] and the Integral Equation Formulation for Scattering (IEFS) [17]. Since these models need prior knowledge of the positions of primary particles within each aggregate (to calculate the optical cross sections) and averaging over orientations is also required to simulate the properties of a randomly-oriented ensemble of aggregates, it is almost impossible to implement these exact models into practical diagnostic techniques. Instead of using these exact models, a simplified model - the Rayleigh-Debye-Gans theory for Fractal Aggregates (RDG-FA), has been widely adopted in various optical diagnostic techniques. RDG-FA is based on the assumption that the primary particles are within the Rayleigh regime $(x<0.5)$ and that the interactions between primary particles can be neglected. Therefore, the absorption cross section of such an aggregate is simply the primary particle absorption cross section (absorption efficiency times the geometric cross sectional area) multiplied by the number of primary particles. The number of primary particles $(N)$ in a fractal aggregate can be calculated from:

$$
N=k_{f}\left(\frac{R_{g}}{a}\right)^{D_{f}}
$$

Where $R_{g}$ is the radius of gyration, $a$ is the primary particle radius, and $k_{f}$ and $D_{f}$ are the fractal prefactor and fractal dimension, which are both used to describe the morphology of the soot aggregates [18]. This theory indicates that the absorption coefficient of the soot aggregate is the same as that of the primary particle so it does not contain any information about the morphology of the soot aggregate, although the overall absorption cross section does depend on morphology, owing to the relationship for the number of primary particles shown in eq. 6 .

A comparison between the scattering and absorption cross sections predicted by RDG-FA and GMM [16], or IEFS [17] shows that RDG-FA predicts reasonably well (< 10\% error) for the absorption cross sections of soot particles encountered in typical hydrocarbon flame conditions.

\subsection{Concepts in CBT-TCS}

This sub-section begins by exploring the use of colour ratios for measurements and then explains in detail why Mie theory is chosen as the scattering model for CBT-TCS.

\subsubsection{Camera concepts}

The value recorded by a digital camera at a given photo site as a result of exposure to the radiation from spherical soot particles can be modelled using the following equation:

$$
R^{\prime}(T, d)=\frac{6 f_{v} V}{d} \cdot \int\left(Q_{a b s}(\lambda, d) \cdot I_{\lambda}(T) \cdot \operatorname{CSR}_{\mathrm{red}}(\lambda)\right) d \lambda
$$

This equation is for the red channel, but similar equations for green and blue are easily written. In this equation, $R$ ' is the calculated red pixel value, $\operatorname{CSR}_{\text {red }}(\lambda)$ is the camera response function for red pixels (see section 3.2 for more details), and all other variables are as previously defined. The presence of alternative sources of radiation in the flame will be shown to be negligible in section 3.5. The flame has implicitly been assumed to be optically thin when writing eq. 7 . This assumption will be checked in section 4.3 .

\subsubsection{Sensitivity of CBT-TCS}

A numerical sensitivity analysis was carried out in order to investigate the reliability of the measurements made using different scattering models for CBT-TCS. The colour ratios R/G and R/B which are important in CBTTCS may be calculated by using equation 7 with a range of temperatures and particle diameters. The sensitivity of the colour ratios to change in these two parameters informs about the precision of CBT-TCS.

Fig. 3 Plot of the diameter/temperature look-up tables when using the Rayleigh-Gans theory 
Fig. 3 shows the sensitivity of the look-up table when using the RG theory (eq. 3). From the two subfigures, it can be seen that both the R/G and R/B ratios are very sensitive to the change of temperature. However, the contour lines are vertical, indicating no sensitivity to particle size. This is because the diameter dependence of the absorption coefficient is cancelled out when a ratio is taken.

Fig. 4 Plot of the diameter/temperature look-up tables when using the Rayleigh-Gans theory with Penndorf extension correlation

Fig. 4 plots look-up table sensitivity when using the Penndorf extension to the RG theory (eq. 4). The contour plots continue to demonstrate that the colour ratios are sensitive to temperature. From the slope in the curves, the sensitivity of the R/B ratio to change in diameter is lower than that of the R/G ratio, but ultimately neither ratio is able to give a high resolution diameter distribution, especially in the temperature range below $1500 \mathrm{~K}$.

Fig. 5 Plot of the diameter/temperature look-up tables when using the Mie theory

The sensitivity of the R/B and R/G ratios when using the Mie theory (eq. 2) is plotted in fig. 5; both the R/G and $\mathrm{R} / \mathrm{B}$ ratios are sensitive to temperature. In addition, the $\mathrm{R} / \mathrm{B}$ ratio is more sensitive to change in diameter than the $\mathrm{R} / \mathrm{G}$ ratio. Because the contours have a turning point, there are now two diameter solutions for a given colour ratio. Therefore, when retrieving particle diameter, an upper limit of approximately $100 \mathrm{~nm}$ must be enforced.

For all tested scattering models, only temperatures above $1400 \mathrm{~K}$ may be measured by the system, because of the turning point in the R/B ratio. However, this is not a significant limitation, because when the temperature is below $1500 \mathrm{~K}$ the raw pixel values are very low (i.e. below 5 counts) since most thermal radiation will be in the infrared, and thus not recorded by this camera.

In conclusion, the temperature field is sensitive to both the $\mathrm{R} / \mathrm{G}$ and $\mathrm{R} / \mathrm{B}$ ratios for all the scattering models. The soot diameter distribution calculated by the Penndorf extension and the Mie theory will have a low resolution, but this is still an improvement on Rayleigh-Gans theory.

Taking the Mie theory to be the reference scattering model, owing to its roots in Maxwell's equations, it can be seen from this data that the RG theory with the Penndorf extension is generally effective in reproducing the features of the Mie theory at reduced computational cost, whereas the RG theory is perhaps a simplification too far. The differences between the contours of the Mie theory and the Penndorf extension become more pronounced as particle size increases, as expected from fig. 1.

\subsubsection{Scattering model for CBT-TCS}

Despite its track record, RDG-FA is not the best choice of scattering model for CBT-TCS. This is because all morphological information embedded in RDG-FA (i.e. $N, R_{g}, k_{f}, D_{f}$ and $d_{p}$ ) is lost when a colour ratio is taken. To demonstrate this, we use eq. 3, 6 and 7, to write the red to green colour ratio for RDG-FA as:

$$
\frac{R^{\prime}}{G^{\prime}}=\frac{\frac{6 f_{v} V}{d} \cdot k_{f}\left(\frac{R g}{d_{p}}\right)^{D_{f}} \cdot \int\left(4 x E(m) \cdot I_{\lambda}(T) \cdot \mathrm{CSR}_{\mathrm{red}}(\lambda)\right) d \lambda}{\frac{6 f_{v} V}{d} \cdot k_{f}\left(\frac{R_{g}}{d_{p}}\right)^{D_{f}} \cdot \int\left(4 x E(m) \cdot I_{\lambda}(T) \cdot \mathrm{CSR}_{\text {green }}(\lambda)\right) d \lambda}
$$

Notice how any terms relating to particle morphology are lost. In contrast, for Mie theory (eq. 2), the ratio remains a function of particle size owing to the fact that the absorption efficiency is a multiple-term function of particle diameter and wavelength.

We justify the adoption of Mie theory instead of RDG-FA on the grounds that it does not significantly affect the derived temperature, and yet allows some limited information about particle size to be recovered.

Fig. 6 Contour plot of temperature derived from Mie theory as a function of R/B and R/G colour ratios using a $60 \mathrm{~nm}$ spherical particle diameter.

Fig. 6 shows how the derived temperature varies with colour ratio when applying the Mie theory to a family of computed black body spectra when assuming spherical particles of $60 \mathrm{~nm}$ diameter. By performing a similar analysis, but replacing the scattering model with the RG/RDG-FA theory and using a primary particle diameter 
of $15 \mathrm{~nm}$, a similar map can be produced. (Reference [16] gives a primary particle diameter of $15 \mathrm{~nm}$ as typical of combustion generated soot, and our measurement shows that $60 \mathrm{~nm}$ is the typical size.

Fig. 7 Temperature difference between Mie theory (60 $\mathrm{nm}$ spherical particle diameter) and RG/RDG-FA theory ( $15 \mathrm{~nm}$ primary particle diameter)

Fig. 7 shows the temperature difference between the Mie theory and the RG/RDG-FA theory. These two figures show that for a realistic flame temperature of less than $2100 \mathrm{~K}$, the difference is below $25 \mathrm{~K}$, which is below our experimental uncertainty $(\geq 30 \mathrm{~K})$ and therefore not significant.

Since the 'diameter' calculated by Mie theory is the diameter of the equivalent spherical particle which has the same absorption characteristics as the soot aggregate, its physical meaning is most similar to the radius of gyration parameter $\left(R_{g}\right)$ in RDG-FA. To test whether the 'diameter' value can be used as a qualitative measurement to the soot aggregate size, its value has been compared to the radius of gyration $\left(R_{g}\right.$ in eq. 6) calculated by RDG-FA theory, using the following process:

1. Calculate the absorption cross sections of soot aggregates across a range of radius of gyration (5$100 \mathrm{~nm}$ ) by using the RDG-FA theory. The morphological parameters were set to values typical of combustion-generated soot aggregates $\left(a=15 \mathrm{~nm}, k_{f}=2.3\right.$, and $D_{f}=1.78$ [16]). The number of primary particles in the soot aggregates ranges from 1 to 230 by using these morphological parameters. The incident wavelength was set to $550 \mathrm{~nm}$.

2. Mie theory was then used to calculate the equivalent 'diameter' value with the same absorption cross section value calculated in step 1 .

3. The radius of gyration was then plotted versus 'diameter' and a curve fitted.

Fig. 8 The correlation between 'diameter' $(D)$ calculated by Mie theory and 'Radius of gyration' $(R)$ in RDG-FA. The solid line is the exponential fitting curve: $R=0.04 D^{1.744}$

The result of this analysis is plotted in fig. 8 . This shows that the Mie-derived 'diameter' is proportional to the RDG-FA radius of gyration parameter to the power of 1.744 . The monotonic relationship between these two parameters indicates that Mie-derived diameter could be a useful indicator of overall particle size, at least for the conditions considered here. This monotonic relationship is valid for all different sizes of soot aggregates so not limited to $100 \mathrm{~nm}$.

To conclude, CBT-TCS adopts Mie theory instead of the established RDG-FA because the derived temperature is the same to within experimental error for typical flame conditions, and yet the substitution of scattering model allows some information about the overall size of the particle aggregate to be recovered from the camera image. The recovered particle size is likely to have limited accuracy, but could still be expected to be of utility, particularly when using this technique for comparative studies.

\subsubsection{Accuracy of Mie theory}

The main scattering model used in CBT-TCS is the Mie theory, which means the soot particles are assumed to be spherical. Though the soot particles in laminar diffusion flames of this kind are known to be fractal aggregates with spherical primary particles, using Mie theory will not noticeably affect the accuracy of the temperature distribution. As shown in section 2.3.2, replacing the RDG-FA theory with the Mie theory to calculate the temperature leads to a temperature deviation of less than $25 \mathrm{~K}$ at realistic flame temperature, which is smaller than the experimental uncertainty.

$\mathrm{Li}$ et al. [19] investigated the numerical accuracy of the spherical approximations when calculating the scattering properties of the soot aggregates by calculating the phase matrix of the soot particles using the generalized Multiple Mie Theory (GMM). They calculated the phase matrix of the soot aggregate, and the spherical soot particle with either equivalent volume or surface area. Their simulation showed that a spherical particle with equivalent volume has a weaker forward scattering and a stronger backscattering compared to the soot aggregate, with an average error in the normalized phase function of around $20 \%$ for incident light of wavelength $628 \mathrm{~nm}$, while the spherical particle with equivalent surface area has stronger forward scattering and weaker backscattering and an average error also around $20 \%$. 
The derived particle size from CBT-TCS is not the physical size of the soot aggregate. Instead, it is the size of equivalent spherical particles with same absorption coefficient as the real soot aggregate. Finally, the absolute soot volume fraction measured by CBT-TCS will be affected by the spherical particle assumption because the absolute thermal radiation is proportional to the total surface area of the soot particles, which will be different for the soot aggregate compared to spherical soot particles. The soot volume fraction measurement by applying more sophisticated scattering models (such as RDG-FA) also remains challenging because the fractal parameters of the soot in the flame vary spatially and must be determined using expensive offline diagnostics and invasive sampling techniques that might themselves influence the morphology.

\subsection{Particulate temperature is the same as local flame temperature}

CBT-TCS assumes that the temperature of the soot particles is equal to the local gas temperature throughout the flame. Many researchers have argued that this assumption is valid. Matsui et al. [20] showed that the particle temperature would respond to changes in the surrounding gas temperature within around $10^{-8} \mathrm{~s}$. They also showed that the particle would achieve a steady-state temperature within $0.3 \mathrm{~K}$ of the surrounding gas temperature. Zhao and Ladommatos [21] summarise previous work and conclude that the temperature difference between soot particles and surrounding gases is below $1 \mathrm{~K}$, providing thermal equilibrium has been attained, which typically happens on a time scale below 1 microsecond. Furthermore, it has been shown that soot-based pyrometry techniques agree within experimental uncertainty with gas-based (e.g. nitrogen) CARS measurements, for example, Hall and Bonczyk [4].

\section{Methodology}

\subsection{Cone Beam Tomographic - Three Colour Spectrometry overview}

In CBT-TCS, a flame is imaged with a colour camera, and the imaging lens is set to a small aperture (i.e. high $\mathrm{f} / \#)$. Each pixel on the camera sensor is associated with one of the primary colours - red, green or blue - and this allows the flame emission to be measured simultaneously in three spectral windows across the sensor. Owing to the small aperture of the imaging lens, each pixel can be thought of as integrating the light emitted along a certain line through the flame (providing the flame is optically thin, see section 4.3 where this is tested). Therefore, the three colour images which result from the analysis of camera data can be used as projections of the colour property field, and a Cone Beam Tomographic (CBT) reconstruction algorithm can be employed to reconstruct a map of colour values at all locations within the flame volume. The 'cone' in this technique refers to the cone of rays passing through the flame which meet at a point that is the optical centre of the imaging lens, and then continue, to terminate on the individual pixels of the camera sensor. This work employs the 3D filtered back-projection algorithm [22] to reconstruct the property field from the cone-beam projections because it is computationally efficient, accurate and readily integrated into MATLAB. Furthermore, by adopting this algorithm, CBT-TCS could be extended in the future to applications which lack cylindrical symmetry, in the same way as other measurement techniques have previously been adapted by researchers [23].

Three Colour Spectrometry (TCS) is a technique where the emission from a flame is interrogated in three spectral windows [21]. Assuming the emission of the flame comes from the thermal radiations of soot particles only (section 3.5) and the attenuation of thermal radiations along the optical path is negligible (section 4.3), and the measured radiation intensity can be characterised - for example, by use of a black body spectrum and a suitable scattering model (scattering models were discussed in section 2.2 and 2.3) - taking ratios (e.g. red/green and red/blue) provides two pieces of information which could in principle be used to provide two measurements. The two physical parameters must affect the shape of the spectrum in order for this to be achieved. For the analysis presented here, the temperature and particle size are recovered (within certain limits of precision and accuracy). Note that this can be achieved without resorting to an absolute calibration of any part of the apparatus.

CBT-TCS combines these two techniques, by taking ratios of the colour values in the reconstructed property field to measure spatially resolved temperature and particle size fields within a flame.

By adding an additional absolute instensity calibration to the camera, soot volume fraction measurements are also derived from the images taken with this system.

This method section will now continue by describing the apparatus before detailing how the data is processed.

\subsection{Experimental realisation}

The overall experimental setup is shown in fig. 9. It consists of a co-annular laminar diffusion flame burner, the air and fuel delivery system, and the imaging system. The co-annular laminar diffusion flame burner is a copy of the burner described by Gülder et al. [8]. The burner consists of a fuel tube of $10.9 \mathrm{~mm}$ inner diameter, 
centred in a $100 \mathrm{~mm}$ diameter air nozzle. To minimize the glare and light reflections from the inner walls of the surrounding chimney, the wall surface is black anodized and only one window (made of fused silica) is used for optical access.

Fig. 9 Schematic of the experimental setup. MFC stands for Mass Flow Controller.

The mass flow rates of air and fuel are accurately controlled by two mass flow controllers (MFCs). An interface control box and a LABVIEW program are used to control and record the flow rates of fuel and air. The analogue input channels are used to log the actual flow rate signals, the analogue output channels are used to control the mass flow rates remotely, and the digital I/O channels are used to fully open or close the mass flow controllers. The ethylene flow rate was $194 \mathrm{~mL} / \mathrm{min}$ and the air flow rate was $200 \mathrm{~L} / \mathrm{min}$.

A Photron ${ }^{\circledR}$ FASTCAM 1024 PCI high speed camera was used to record the flame image. The maximum resolution is $1024 * 1024$ pixels with a $17.0 \mu \mathrm{m}$ pixel width (corresponding to $23.0 \mu \mathrm{m}$ in the object plane in the current optical setup). The exposure duration was $16 \mathrm{~ms}$. The image can be saved in the 'Bayer' format which only records the true colour channel value for each pixel without any on-board processing such as colour demosaicing. Light from a tungsten lamp was passed through a Hilger \& Watts monochromator which was tuned in increments of $10 \mathrm{~nm}$ in order to determine the relative response of the camera between 400-800 nm. The emission from the monochromator at each setting was verified against a factory-calibrated Ocean Optics USB4000 optical fibre-coupled spectrometer, and the camera response normalised by these measurements. The measured camera response curve is shown in fig. 10.

Fig. 10 Camera sensor response curve (the two different green pixels responses are found in the raw image, and this can be attributed to misalignment of the colour filter array and the CMOS sensor).

The camera sensor has also been calibrated for absolute intensity against the USB4000 spectrometer by alternately placing the camera sensor and then the spectrometer's optical fibre in the imaging plane of a lens system, when a uniformly illuminated screen was being imaged. The 'sensitivity factor', $s$, is as follows:

$$
s=\int\left(I_{\text {lamp }} \cdot \operatorname{CSR}_{\text {red }}(\lambda)\right) d \lambda \div R_{\text {ref }}
$$

Where $I_{\text {lamp }}$ is the measured absolute spectrum from the Ocean Optics spectrometer, and $R_{\text {ref }}$ is the red pixel value measured from the same region in the camera image as the fibre tip position. (Similar equations for the green and blue channels exist, and the average of all three was used for the final value of $s$.)

The average pixel values among 100 flame images are used during experiments. A half-cone pointer was inserted into the fuel pipe to represent the flame and find the focal plane. Because the image definition will degrade significantly outside the depth of field of the optical system, a depth of field calculation with the lensmaker's formula and an assumed flame thickness of $10 \mathrm{~mm}$ led to an aperture selection of $f / 32$. This aperture strongly attenuates most lens aberrations but does begin to introduce undesirable blurring caused by diffraction. The distance between the flame and the optical centre of the lens system (i.e. the vertex point in the cone beam system) $(u)$ and that between the optical centre to the camera detector $(v)$ should be set at $23.6 \mathrm{~cm}$ and $17.4 \mathrm{~cm}$ respectively, so that the minimally-blurred image integrated through the whole flame along an arbitrary optical path will be contained within three pixels without noticeable degradation of definition. The optical system consists of a $50 \mathrm{~mm}$ f/1.8 Nikon lens, a $2 x$ tele/macro convertor, and a LEE 281 optical filter (this attenuated the red and green wavelengths, so that the red, green and blue pixels had comparable values). All these optical components have been spectrally tested to obtain their transmission efficiencies $\left(T_{i}\right)$. Moreover, the radius of the aperture in the 50mm lens $(r)$ has been measured to calculate the collection efficiency $(\eta)$ by eq. (10):

$$
\eta=\pi r^{2} /\left(4 \pi u^{2}\right)
$$

In the current optical setup, the radius of the aperture is $1.46 \mathrm{~mm}$, and the radiance received by the camera detector $\left(I_{\lambda}^{\prime \prime}\right)$ is:

$$
I_{\lambda}^{\prime \prime}=\left(\prod_{i} T_{i}\right) \cdot M^{-2} \cdot \eta \cdot \frac{6 f_{v} V}{d} \cdot Q_{a b s} \cdot I_{\lambda}(T)
$$

where $M=v / u$ is the optical magnification. 


\subsection{Three Colour Spectrometry}

A software package written in MATLAB with a GUI is used to post-process the radiance recorded by the camera. It integrates many functions, including the colour demosaicing, the 3D cone beam tomography, the 3D median filtering, the calculation of look-up tables used in the three colour spectrometry, the mapping of the colour ratios to field properties (temperature, soot diameter and soot volume fraction), and circumferential averaging for axisymmetric objects.

Fig. 11 The signal processing strategy used in the modified three colour spectrometry method.

In order to derive the TCS measurements of temperature and diameter efficiently, a pair of look up tables are generated which relate the red/green $(\mathrm{R} / \mathrm{G})$ and red/blue (R/B) ratios to the physical parameters. Fig. 11 (step 1) illustrates the look up table calculation process, which proceeds as follows: Camera pixel values are calculated for a range of temperatures and particle sizes using equation 7 yielding values for $R^{\prime}$, the calculated red pixel value, green value $G^{\prime}$, and blue value, $B^{\prime}$. The ratios $R^{\prime} / G^{\prime}$ and $R^{\prime} / B^{\prime}$ are then calculated, and the relationship to the input $T$ and $d$ stored.

In order to derive temperature and particle diameter for any given location in the flame, we follow the steps shown in fig. 11 (step 2). From the camera image, we apply the tomographic algorithm to reconstruct R, G and $\mathrm{B}$ values for each location in the volume, and then the measured ratios $R / G$ and $R / B$ are calculated at all positions. Conceptually, for each position in turn, the correct contour line can then be chosen from the two look up tables (e.g. fig. 5), and the location where the two contours cross provides the result in $T$ and $d$.

Note that the act of taking the ratio (i.e. $R / G$ and $R / B$ ) causes the soot volume fraction to be cancelled out. Indeed, the theory employed to model the emission spectrum from the sooting flame in this analysis has no mechanism to allow a varying soot volume fraction to affect the spectral signature of the flame. In order to take soot volume fraction measurements from the flame, the camera sensor has been calibrated for absolute response to incoming radiation as previously described. This calibration provides a 'sensitivity factor', $s$, which relates the calculated pixel response (from eq. 3, e.g. $R^{\prime}$ ) to the measured pixel response (e.g. $R$ ). Soot volume fraction is then determined following fig. 10 (step 3) using:

$$
f_{v}=s \cdot \frac{1}{3} \cdot\left(\frac{R}{R \prime \prime}+\frac{G}{G \prime \prime}+\frac{B}{B \prime \prime}\right)
$$

Where $R$ is a measured pixel value, and $R$ ' ' is the calculated pixel value from eq. 3 with $f_{v}$ set equal to unity.

In summary, the CBT-TCS technique is able to determine spatially resolved temperature and diameter fields provided that the camera has been spectrally calibrated. If the camera has an additional absolute calibration, then the soot volume fraction may also be determined.

\subsection{Image processing algorithm}

The data analysis which is at the heart of the CBT-TCS technique was optimised by carefully choosing how to implement certain steps.

1. The noise in the reconstructed field produced by the cone beam algorithm was significantly reduced by down-sampling the source image by a factor of 2 . This level of down-sampling balanced the need to increase the difference between adjacent paths above the noise level of the image, without causing undue loss of detail. The resulting voxel (volumetric pixel) size at this down-sampling ratio is $(46 \mu \mathrm{m})^{3}$.

2. The algorithm employed in our analysis system allows for objects that lack any symmetry properties to be reconstructed, given enough projections (i.e. views of the object from different angles). In our case, the object is axisymmetric so we supplied the same projection $n$ times. The reconstructed temperature field began converging on a self-consistent solution for $n$ larger than 60. Our analysis employs $n=180$, owing to the minimal increase in computational time.

3. The vectors supplied to the filtered back-projection algorithm were zero-padded up to 8192 samples in conjunction with a Hamming window in order to resolve extra detail at low frequencies, causing smoother profiles.

4. Thresholding was employed to mask out any voxels with a red value below 0.1 , because such low values would cause unreliable ratios.

5. A three-dimensional median filter of size $4 \times 4 \times 4$ pixels was applied to the reconstructed field, to remove outliers. 
6. Whilst the camera was carefully aligned to match a pointer placed in the fuel tube of the burner, the centre of the flame was ultimately measured from the flame image, to account for the positioning uncertainty of a few pixels. Inaccuracy here produces a significant artefact.

\subsection{Interferences with soot luminosity}

Besides the gaseous products and the particles, there are electronically excited radicals created during the oxidation reactions which also emit radiation. Rather than a broadband emission, these radicals emit in discrete spectral regions, an effect called chemiluminescence. In order to investigate whether the emission from these radicals interferes with the broadband emission from the soot locally, the optical fibre coupled into a spectrometer was traversed across an image of the flame created with the standard lens system previously described. The ethylene flow rate was set to $145 \mathrm{~mL} / \mathrm{min}$ and the air flow rate was set to $42 \mathrm{~L} / \mathrm{min}$ to produce a sooting laminar diffusion flame. The optical fibre sampled a circular area with a diameter of about $1 \mathrm{~mm}$ from the flame. Measurements were taken at four different heights: $3 \mathrm{~mm}, 10 \mathrm{~mm}, 20 \mathrm{~mm}, 30 \mathrm{~mm}$ and $40 \mathrm{~mm}$ above the fuel tube. The results are shown in fig. 12.

Fig. 12 Radiation from different parts of an ethylene/air laminar diffusion flame detected by a spectrometer (Fig. 12a: $3 \mathrm{~mm}$ above the fuel tube; Fig. 12b: $10 \mathrm{~mm}$ above the fuel tube) Note that the colour camera can only record light between 400-700 $\mathrm{nm}$.

As shown in fig. 12, at the bottom of the flame (Fig. 12a $-3 \mathrm{~mm}$ above fuel tube), a series of well-defined peaks attributable to radical species such as $\mathrm{OH}, \mathrm{CH}$ and $\mathrm{C}_{2}$ are visible in the measured spectrum in both the middle and edge part. Fortunately, this part of the flame is unlikely to be measured by CBT-TCS, simply because the flame luminosity is far too low to record a reliable signal. Looking instead at higher positions of the flame (Fig. $11 \mathrm{~b}-10 \mathrm{~mm}$ above fuel tube), the spectrum becomes continuous in character and much higher in magnitude, with no observable narrow peaks indicating that chemiluminescence is negligible compared to the flame luminosity. The spectra at $20 \mathrm{~mm}, 30 \mathrm{~mm}$, and $40 \mathrm{~mm}$ have similar shapes as at $10 \mathrm{~mm}$ but higher energy levels. Overall, it is possible that some regions of the flame could see some interference from chemiluminescence, but in this work CBT-TCS was only applied when the flame luminosity was high (maximum integration time is $1 / 60$ second), making interference from radical species unlikely.

The main products generated during the combustion of hydrocarbon fuels are $\mathrm{CO}_{2}$ and water vapour. The emission bands of $\mathrm{CO}_{2}$ and water vapour are centred in the infra-red region [24], which will not be recorded by the camera. Therefore, their interference can be neglected.

When considering sooting flames, the effects of soot precursors - polycyclic aromatic hydrocarbons (PAH) must be considered. Absorption spectra for most simple PAH molecules demonstrate absorption features in the ultraviolet except for a few very large PAH molecules in the lower end of the visible spectrum, such as terrylene $\left(\mathrm{C}_{30} \mathrm{H}_{16}\right.$ has 450 to $600 \mathrm{~nm}$ substructure $)$ and quaterrylene $\left(\mathrm{C}_{40} \mathrm{H}_{20}\right.$ has 500 to $600 \mathrm{~nm}$ substructure $)[25,26]$. PAH ions can be strong absorbers in the visible spectrum $[26,27]$, but their concentrations $(\sim \mathrm{ppt})$ are much lower than neutral PAH species $(\sim \mathrm{ppm})$ [27]. The PAH family has many members which may limit the generality of this simple review, but since the wavelength range covered by the Photron camera is from 400-750 $\mathrm{nm}$ and PAH concentrations reduce with the increase in their molecular mass [28], the impact of molecular and ion PAH should be small.

It can be concluded that the effects of the emissions and absorption by the combustion gases are well controlled. A more detailed review of flame radiation is presented by Tien and Lee [29], who also concluded that the effect of chemiluminescence from intermediate radicals can be neglected in the main part of the flame.

\section{Results and discussions}

\subsection{Temperature, soot diameter and soot volume fraction}

A pure ethylene laminar diffusion flame as detailed in section 3 was used to demonstrate CBT-TCS. Fig. 13 shows the temperature, soot diameter and soot volume fraction profiles in the central axis plane computed by using the Mie theory.

Fig. 13 Temperature, soot diameter and soot volume fraction distributions at the central axis plane calculated by using the Mie theory 
Fig. 13 shows that the soot volume fraction distributions peaks near the edge of the flame, where we believe to be the main sites for pyrolysis reactions since it meets the conditions of high temperature and relatively low oxygen level. The soot particles present in the middle of the flame are mostly diffused from the reaction zone so their concentrations are much lower. Soot particle size and soot volume fraction appears to generally reduce towards the tip of the flame, which could be indicative of soot particle oxidation becoming increasingly important above $z=40 \mathrm{~mm}$.

\subsection{Experimental limitations}

The reconstructed fields shown in fig. 14 exhibit some high spatial frequency noise despite the measures described in section 3.3. Whilst the downsampling step improves the quality of the reconstruction considerably, it is ultimately limited by the precision of the camera. Fig. 14 shows that the typical reconstructed colour distributions are smooth but the colour ratio distributions show 'bumps', especially when the pixel count is very low (regions close to centre). This is because the limited bit depth of the camera cannot distinguish small light intensity differences. Therefore, the noise is expected to be reduced by using a higher precision camera. In the case of the particle diameter map, this noise is exacerbated by the limited discrimination of the technique.

Fig. 14 Typical reconstructed radial colour and colour ratio distributions (in camera pixel count). (3*RG: tripled Red-to-Green ratio; RB: Red-to-Blue ratio)

\subsection{Opacity}

CBT-TCS makes the assumption that attenuation of the emitted radiation along the optical path is negligible due to both scattering and absorption from downstream particles, or, stated another way, that the flame is opticallythin. Adoption of this assumption allows the total emission of the soot particles inside each voxel to be calculated by multiplying the emission of a single particle with the number density - neglecting multiplescattering phenomena. Whether this assumption is valid or not depends on the soot size and concentrations in the flame. In most combustion conditions, the soot volume fraction is small (on the order of ppm), which produces a low optical thickness. Fig. 15 shows the optical thickness at different heights for the flame described in section 4.1. The optical thickness is calculated according to the Beer-Lambert law and the extinction cross section is calculated according to the Mie theory for $600 \mathrm{~nm}$ incident light using measured soot volume fractions and soot particle diameters. The results show that the maximum light loss is around $10 \%$ in the middle part of the flame and the average light loss is around $5 \%$.

\section{Fig. 15 Optical thickness and transmission at different height}

The error caused by the optically-thin approximation can be partially corrected by an iterative method introduced by $\mathrm{Lu}$ et al. [7]. However, the assumption that the attenuation term is a constant in the method introduced by $\mathrm{Lu}$ et al. can introduce some error because the attenuation actually depends on the position of the voxels inside the flame and the particle distribution along the optical path.

\subsection{Uncertainty analysis}

An uncertainty analysis has been undertaken in order to characterise the precision of the measurements taken by CBT-TCS. Fig. 16 consists of three plots which illustrate in turn flame instability, temperature precision and soot volume fraction uncertainty. Fig.16 (a) is produced by finding the coefficient of variation (COV) at each point in the image recorded by the camera by computing the mean and standard deviation at each pixel location through the series of 100 recorded images. At a height of $30 \mathrm{~mm}$, the flame luminosity reaches a maximum, and the noise created by the camera is least significant. Below this point, the signal from the flame becomes steadily lower and the COV worsens. Above $30 \mathrm{~mm}$, the signal decreases and flame 'flutter' becomes more of a problem, causing up to $8 \% \mathrm{COV}$ at the flame tip. These fluctuations are amplified throughout the data analysis and lead to height dependence in plots (b) and (c). Fig. 16 (b) shows that the best precision for CBT-TCS is around $1.5 \%$ at a height of $30 \mathrm{~mm}$ where the flame is very stable. The measurement uncertainty is the worst at the flame tip and is around $8 \%$, mainly because of the instability of the flame. Fig. 16 (c) shows the uncertainty in the derived soot volume fraction, which varies from $40-80 \%$. The precisions of the measurements of temperature and soot volume fraction are temperature dependent because of the nonlinear correlation between the thermal radiation and temperature.

Fig. 16 (a) COV of the measured light intensity formed from a sequence of 100 images, (b) uncertainty in the calculated temperature and (c) uncertainty in the calculated soot volume fraction. Four data series represent different heights in the flame. 


\subsection{Comparisons}

As the Gülder burner employed in this work is a reference design, it has been investigated by others. The results from CBT-TCS are compared to the data reported by Snelling et al. [5] in fig. 17. Snelling et al. report temperature measurements of this flame using Coherent Anti-Stokes Raman Scattering spectroscopy (CARS) and a 2D tomographic soot spectrometry technique. Spatial soot distributions have been measured using 2D line-of-sight light attenuation (LOSA) and 2D tomographic soot spectrometry. The precision of the CARS measurement was estimated at around $\pm 25-50 \mathrm{~K}$ at the flame height of $30 \mathrm{~mm}$ [5]; the precision of the 2D LOSA was estimated at around $20-30 \%$ and that of the 2D tomographic soot spectrometry was around $35-45 \%$ [30]. The ethylene flow rate in their experiment is also $194 \mathrm{~mL} / \mathrm{min}$, but the air flow rate without a chimney is $284 \mathrm{~L} / \mathrm{min}$, which is higher than our current experimental setup. The reason our data employs a lower air flow rate is that the flame becomes noticeably unstable for a flow rate higher than $200 \mathrm{~L} / \mathrm{min}$. The chimney we used to isolate the flame from air currents in the laboratory constrains the air flow and may be responsible for this difference. This mismatch may also be responsible for a small change in flame height and width, which may be the cause of some of the differences which are visible in fig. 16. In all cases, the error bars on the CBT-TCS data follow the values presented in fig. 16.

Fig. 17 Comparison of the temperature and soot volume fraction distributions against those measured by Snelling et al. [5] using LOSA (line-of-sight attenuation); SST (2D tomographic soot pyrometry); CARS (Coherent Anti-Stokes Raman Spectroscopy). CBT-TCS: Cone-Beam Tomographic Three Colour Spectrometry is the data reported here.

In general, fig. 17 shows that the radial profiles of both temperature and soot volume fraction at heights of 10 , 20,30 and $50 \mathrm{~mm}$ are in good agreement. The differences in the values are largely within the measurement uncertainty. However, the uncertainty in the top region of the flame is much higher than elsewhere, and this is mainly due to the instability of the flame as discussed above. The oscillatory pattern seen most clearly at the 30 $\mathrm{mm}$ height is thought to be due to the limited dynamic range of the 10-bit CCD sensor which was used to capture the data, since the absolute pixel values at low flame radius are changing by a value which is barely above the digitisation limit (see section 4.2). At the edges of the flame, where the luminosity is higher, the CBTTCS data is stable.

\section{Conclusions}

The principles of and experimental setup for a newly-developed cost-effective optical diagnostic technique, called Cone Beam Tomographic Three Colour Spectrometry (CBT-TCS), to measure the spatially distributed temperature, soot diameter, and soot volume fraction has been introduced in detail.

Compared to existing non-laser based multi-wavelength absorption/extinction spectroscopy techniques used to measure the temperature and soot volume fraction in combustion systems, this technique can measure the temperature, soot diameter and soot volume fraction distributions in a single shot, by using a calibrated camera system. Existing techniques are generally limited to measuring point-based or planar (applying 2D tomography technique) temperature and soot volume fraction distributions in a single shot. The laser based measurement techniques, such as Laser-Induced Incandescence (LII), can achieve a better signal-to-noise ratio, but are usually point-based measurement for the soot volume fraction and primary particle size.

The data analysis relies on the adoption of Mie theory and the underlying assumption of spherical soot particles. We have demonstrated that this is of little concern for temperature measurement, and an analysis of the CBTTCS look-up tables shows that temperature is very sensitive to colour ratio value. We concede that particle size is measured with considerably lower precision, and that the recovered particle size should only be used a guide to the overall size of a particular soot aggregate. Nonetheless, this information is useful, particularly in comparative studies. With the extra absolute calibration available on our camera, the measurement of soot volume fraction was possible.

The technique has been applied to an ethylene fuelled laminar diffusion flame burner. An uncertainty analysis shows that the uncertainty in both the temperature and soot volume fraction measurements are temperature and height dependent and they become much higher at the top of the flame, mainly because of flame instability. In 
the reaction region, the flame is very stable and the precision in the temperature measurement ranges from $1.5 \%$ to $3 \%$ and the precision in the soot volume fraction measurement ranges from $35 \%$ to $45 \%$.

The experimental data have also been compared to the results reported by Snelling et al. [5] because they used the same burner with the same fuel flow rate. The comparison shows consistent temperature and soot volume fraction profiles at different flame heights, with differences generally within the uncertainty of the measurements. Compared to CARS, CBT-TCS can achieve a comparable precision $(\sim 2 \%)$ but the measurable temperature range is more limited because thermal radiation shifts into the infra-red region at lower temperatures, out of range of the camera and optics. The soot volume fraction measured by CBT-TCS has poorer precision compared to 2D LOSA (20-30\%) but has comparable precision to the reported 2D tomographic soot spectrometry $(35-45 \%)$. The high uncertainty in the soot volume fraction measurement is due to the nonlinear relationship between the temperature and thermal radiation.

In summary, CBT-TCS is an effective technique for measuring the temperature and soot loading within a laminar diffusion flame, with sufficient precision to rival competitive techniques with greater convenience and lower cost. Some information regarding average soot particle size may also be retrieved. In the future, CBTTCS could also be applied to asymmetric flames by taking multiple simultaneous projections.

\section{References}

[1] Hottel HC, Broughton FP. Determination of true temperature and total radiation from luminous gas flames. Ind. Eng. Chem. Anal. Ed. 1932; 4; 166-175.

[2] Hulst HC. Light scattering by small particles. New York: Wiley; 1957.

[3] Mie G. Beiträge zur Optik trüber Medien, speziell kolloidaler Metallösungen. Analen der Physik 1908; 330; 377-445.

[4] Hall RJ, Bonczyk PA. Sooting flame thermometry using emission/absorption tomography. Appl. Opt. 1990; $29 ; 4590-4598$.

[5] Snelling DR, Thomson KA, Smallwood GJ, Gülder OL, Weckman EJ, Fraser RA. Spectrally resolved measurement of flame radiation to determine soot temperature and concentration. AIAA journal 2002; 40; 1789-1795.

[6] AyrancI I, Vaillon R, Selçuk N, André F, Escudié D. Determination of soot temperature, volume fraction and refractive index from flame emission spectrometry. J. Quant. Spectrosc. Radiat. Transfer 2007; 104; $266-276$.

[7] Lu J, Lou C, Zhou H. Experimental investigation on soot volume fraction in an ethylene diffusion flame by emission spectrometry without optically-thin assumption. J. Phys.: conference series 2009; 147; 012084.

[8] Gülder OL, Snelling DR. Influence of nitrogen dilution and flame temperature on soot formation in diffusion flames. Combust. Flame 1993; 92; 115-124.

[9] Kattawar GW, Eisner M. Radiation from a homogeneous isothermal sphere. Appl. Opt. 1970; 9; 26852690.

[10] Bohren CF, Huffman D. Absorption and scattering of light by small particles. New York: Wiley; 1983.

[11] Penndorf RB. Scattering and extinction coefficients for small absorbing and nonabsorbing aerosols. J. Opt. Soc. Am. 1962; 52; 896-902.

[12] Coderre AR, Thomson KA, Snelling DR, Johnson MR. Spectrally resolved light absorption properties of cooled soot from a methane flame. Appl. Phys. B 2011; 104; 175-188.

[13] Migliorini F, Thomson KA, Smallwood GJ. Investigation of optical properties of aging soot. Appl. Phys. B 2011; 104; 273-283.

[14] Smyth KC, Shaddix CR. The elusive history of $m=1.57-0.56 i$ for the refractive index of soot. Combust. Flame 1996; 107; $314-320$.

[15] Sorensen CM. Light scattering by fractal aggregates: a review. Aerosol Sci. Technol. 2001; 35; 648-687.

[16] Liu F, Smallwood GJ. The effect of particle aggregation on the absorption and emission properties of mono- and polydisperse soot aggregates. Appl. Phys. B. 2011; 104; 343-355.

[17] Farias TL, Koylu UO, Carvalho MG. Range of validity of the Rayleigh-Debye-Gans theory for optics of fractal aggregates. Appl. Opt. 1996; 35; 6560-6567.

[18] Jullein R and Botet R. Aggregation and fractal aggregates. Singapore: World Scientific; 1987.

[19] Li H, Liu C, Bi L, Yang P, Kattawar GW. Numerical accuracy of 'equivalent' spherical approximations for computing ensemble-averaged scattering properties of fractal soot aggregates. J. Quant. Spectrosc. Radiat. Transfer. 2010; 111; 2127-2132.

[20] Matsui Y, Kamimoto T, Matsuoka S. A study on the application of the two color method to the measurement of flame temperature and soot concentration in diesel engines. SAE, Detroit, 1980. 
[21] Zhao H, Ladommatos N. Optical diagnostics for soot and temperature measurement in diesel engines. Prog. Energ. Combust. 1998; 24; 221-255.

[22] Feldkamp LA, Davis LC, Kress JW. Practical cone-beam algorithm. J. Opt. Soc. Am. A 1984; 1; 612-619.

[23] Darrell A, Meyer H, Marias K, Brady M, Ripoll J. Weighted filtered backprojection for quantitative fluorescence optical projection tomography. Phys. Med. Biol. 2008; 53; 3863-3881.

[24] Wassel AT, Edwards DK. Molecular gas radiation in a laminar or turbulent pipe flow. J. Heat Transfer 1976; 98; 101-107.

[25] Du H, Fuh RA, Li J, Corkan LA, Lindsey JS. PhotochemCAD: a computer-aided design and research tool in photochemistry. Photochem. Photobiop. 2008; 68; 141-142.

[26] Ruiterkamp R, Halasinski T, Salma F, Foing BH, Allamandola LJ, Schmidt W, Ehrenfreund P. Spectroscopy of large PAHs: laboratory studies and comparison to the Diffuse Interstellar Bands. Astron. Astrophys. 2002; 390; 1153-1170.

[27] Biennier L, Salama F, Gupta M, O’Keefe A. Multiplex integrated cavity output spectroscopy of cold PAH cations. Chem. Phys. Lett. 2004; 387; 287-294.

[28] Weilmunster P, Keller A, Homann KH. Large molecules, radicals, ions, and small soot particles in fuelrich hydrocarbon flames part i: positive ions of polycyclic aromatic hydrocarbons (PAH) in low-pressure premixed flames of acetylene and oxygen. Combust. Flame. 1999; 116; 62-83.

[29] Tien CL, Lee SC. Prog. Flame radiation. Energy Combust. Sci. 1982; 8; 41-59.

[30] Thomson KA, Gülder OL, Weckman EJ, Fraser RA, Smallwood GJ, Snelling DR. Soot concentration and temperature measurements in co-annular, nonpremixed $\mathrm{CH} 4$ /air laminar flames at pressures up to $4 \mathrm{MPa}$. Combust. Flame. 2005; 140; 222-232. 


\section{Appendix A: uncertainty analysis}

The uncertainty of the measurement performed by the camera itself can be quantified by the coefficient of variation $\left(\mathrm{COV}, C V_{x}\right)$ of the pixel intensity. $\mathrm{COV}$ is defined as the standard deviation $\left(\mathrm{SD}, S_{x}\right)$ divided by the mean value $(\bar{x})$, and is thus a normalised measure of fluctuations. Since the uncertainty of the light intensity measured by the camera is due to the instability of the flame and the intrinsic noise of the camera detector (which is the same for different colour channels), the COV for different colour channels $\left(C V_{R}, C V_{G}, C V_{B}\right)$ differ only because the red and green channels detect more light than the blue channel. The colour ratios ( $R G$ : red to green, $R B$ : red to blue) are used to measure the temperature and soot diameter. The COV of the colour ratios $\left(C V_{R G}, C V_{R B}\right)$ are calculated as:

$$
C V_{R G}=\sqrt{\left(\left.\frac{\partial(R / G)}{\partial R}\right|_{\bar{R}, \bar{G}} \cdot S_{R}\right)^{2}+\left(\left.\frac{\partial(R / G)}{\partial G}\right|_{\bar{R}, \bar{G}} \cdot S_{G}\right)^{2}} /(\bar{R} / \bar{G})=\sqrt{C V_{R}^{2}+C V_{G}^{2}}
$$

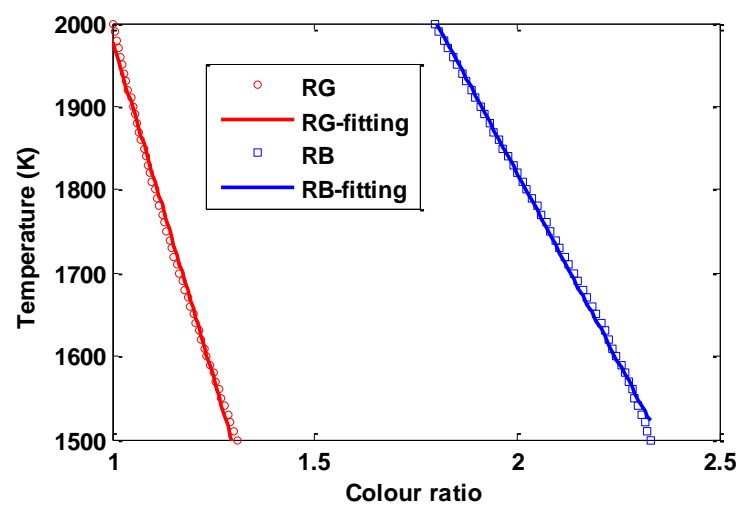

Fig. A1 Temperature as a function of colour ratio. Look up table values are the plotted points, and the line is a linear best fit, which can be seen to closely mimic the trend. (A soot diameter of $50 \mathrm{~nm}$ is assumed here.)

The response of temperature to the colour ratios in the look-up table is very close to linear. This relationship is shown in fig. A1. The temperature can therefore be calculated as:

$$
T=\left(T_{1}+T_{2}\right) / 2=\left(\left(m_{1} \cdot R G+c_{1}\right)+\left(m_{2} \cdot R B+c_{2}\right)\right) / 2
$$

Since both colour ratios are used to calculate the temperature, the standard deviation of temperature is calculated by:

$$
S_{T}=\sqrt{S_{T 1}^{2}+S_{T 2}^{2}} / 2=\sqrt{\left(m_{1} \cdot S_{R G}\right)^{2}+\left(m_{2} \cdot S_{R B}\right)^{2}} / 2
$$

According to Planck's thermal radiation law, the spectral radiance $\left(I_{\lambda}\right)$ is calculated by the formula:

$$
I_{\lambda}=A \cdot f_{v} \cdot \varepsilon(d, \lambda) \cdot(\exp (B / \lambda T)-1)^{-1}
$$

where $A$ and $B$ are two constants, $f_{v}$ is the soot volume fraction, $\varepsilon$ is the emissivity of a single soot particle, $d$ is the soot diameter and $\lambda$ is the wavelength. The sensitivity analysis shows that the emissivity is not very sensitive to the diameter or wavelength and under typical flame temperature the exponential term is much larger than 1 . The COV of the theoretical thermal radiation at a specific wavelength for unit soot volume fraction is:

$$
C V_{I_{\lambda}}=\left(\frac{d I_{\lambda}}{d T}\right)_{\bar{T}} \cdot \frac{S_{T}}{\bar{I}_{\lambda}}=B \cdot S_{T} /\left(\lambda \cdot \bar{T}^{2}\right)
$$

The soot volume fraction is calculated by taking the ratio between the measured light intensity $\left(I_{\lambda}^{\prime}\right)$ to the theoretical thermal radiation for unit soot volume fraction. Taking into account the spectral response of the camera $\left(R S_{\lambda}\right)$, the transmission efficiencies of the lens and filter $\left(T_{i, \lambda}\right)$, and the sensitivity factor of the camera detector $(S F)$ the soot volume fraction is:

$$
f_{v, \lambda}=I_{\lambda}^{\prime} / I_{\lambda}=I_{\lambda}^{\prime} /\left[\left(S F \cdot A^{\prime} \cdot \exp (-B / \lambda T) \cdot R S_{\lambda} \cdot \Pi T_{i, \lambda}\right]\right.
$$


where $A^{\prime}$ is a constant. We now assume the COV of the soot volume fraction $\left(C V_{f_{v}}\right)$ across the visible spectrum is close to the value at $500 \mathrm{~nm}$ wavelength. $C V_{f_{v}}$ measured by using the red pixel value is:

$$
C V_{f_{v}^{R}}^{2}=C V_{R}^{2}+C V_{S F}^{2}+C V_{I}^{2}+C V_{R S}^{2}+\sum C V_{T_{i}}^{2}
$$

The soot volume fraction value is taken as the average value of the three independent colour channels.

$C V_{f_{v}}=\sqrt{C V_{R}^{2}+C V_{G}^{2}+C V_{B}^{2}+3 \cdot\left(C V_{S F}^{2}+C V_{R S}^{2}+C V_{I}^{2}+\sum C V_{T_{i}}^{2}+\left(B \cdot S_{T} /\left(\lambda \cdot \bar{T}^{2}\right)\right)^{2}\right) / 3}$ eq. (A.8)

$C V_{S F}$ and $C V_{R S}$ are estimated at around 3\% each, mainly due to the instability of the calibration light source. The overall COV of the transmission test is estimated at around $2 \%$, owing to limitations of the spectrophotometer used to take the measurement. 


\section{List of figures}

Fig. 1 Comparison of scattering models (RG in the legend stands for Rayleigh-Gans theory). Top left: the variation of absorption efficiency as a function of size parameter $(x=\pi d / \lambda)$. Bottom left: deviations from Mie theory predictions. Top right: the relative strength of scattering to absorption, which shows that scattering becomes more important as the size parameter increases. Bottom right: particle size parameter versus particle diameter and incident wavelength, used to understand the other three plots in terms of physical parameters.

Fig. 2 Effect of refractive index on absorption efficiency when using the Mie theory

Fig. 3 Plot of the diameter/temperature look-up tables when using the Rayleigh-Gans theory

Fig. 4 Plot of the diameter/temperature look-up tables when using the Rayleigh-Gans theory with Penndorf extension correlation

Fig. 5 Plot of the diameter/temperature look-up tables when using the Mie theory

Fig. 6 Contour plot of temperature derived from Mie theory as a function of $R / B$ and $R / G$ colour ratios using a $60 \mathrm{~nm}$ spherical particle diameter.

Fig. 7 Temperature difference between Mie theory (60 $\mathrm{nm}$ spherical particle diameter) and RG/RDG-FA theory $(15 \mathrm{~nm}$ primary particle diameter)

Fig. 8 The correlation between 'diameter' $(D)$ calculated by Mie theory and 'Radius of gyration' $(R)$ in RDG-FA. The solid line is the exponential fitting curve: $R=0.04 D^{1.744}$

Fig. 9 Schematic of the experimental setup. MFC stands for Mass Flow Controller

Fig. 10 Camera sensor response curve (the two different green pixels responses are found in the raw image, and this can be attributed to misalignment of the colour filter array and the CMOS sensor).

Fig. 11 The signal processing strategy used in the modified three colour pyrometry method

Fig. 12 Radiation from different parts of an ethylene/air laminar diffusion flame detected by a spectrometer (Fig 10a: $3 \mathrm{~mm}$ above the fuel tube; Fig 10b: $10 \mathrm{~mm}$ above the fuel tube) Note that the colour camera can only record light between $400-700 \mathrm{~nm}$.

Fig. 13 Temperature, soot diameter and soot volume fraction distributions at the central axis plane calculated by using the Mie theory

Fig. 14 Typical reconstructed radial colour and colour ratio distributions (in camera pixel count). (3*RG: tripled Red-to-Green ratio; RB: Red-to-Blue ratio)

Fig. 15 Optical thickness and transmission at different height

Fig. 16 (a) COV of the measured light intensity formed from a sequence of 100 images, (b) uncertainty in the calculated temperature and (c) uncertainty in the calculated soot volume fraction. Four data series represent different heights in the flame.

Fig. 17 Comparison of the temperature and soot volume fraction distributions against those measured by Snelling et al. [5] using LOSA (line-of-sight attenuation); SST (2D tomographic soot pyrometry); CARS (Coherent Anti-Stokes Raman Spectroscopy). CBT-TCS: Cone-Beam Tomographic Three Colour Spectrometry is the data reported here.

Fig. A1 Temperature as a function of colour ratio. Look up table values are the plotted points, and the line is a linear best fit, which can be seen to closely mimic the trend. (A soot diameter of $50 \mathrm{~nm}$ is assumed here.) 

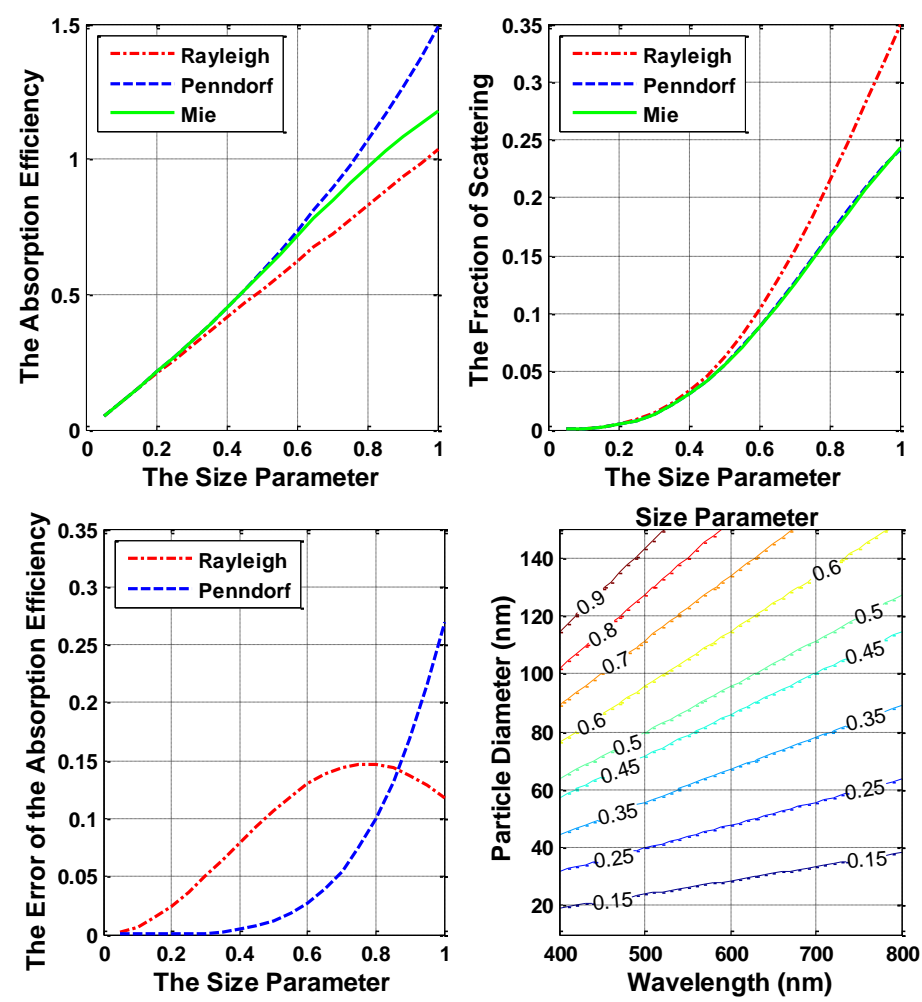

Fig. 1 Comparison of scattering models (RG in the legend stands for Rayleigh-Gans theory). Top left: the variation of absorption efficiency as a function of size parameter $(x=\pi d / \lambda)$. Bottom left: deviations from Mie theory predictions. Top right: the relative strength of scattering to absorption, which shows that scattering becomes more important as the size parameter increases. Bottom right: particle size parameter versus particle diameter and incident wavelength, used to understand the other three plots in terms of physical parameters.

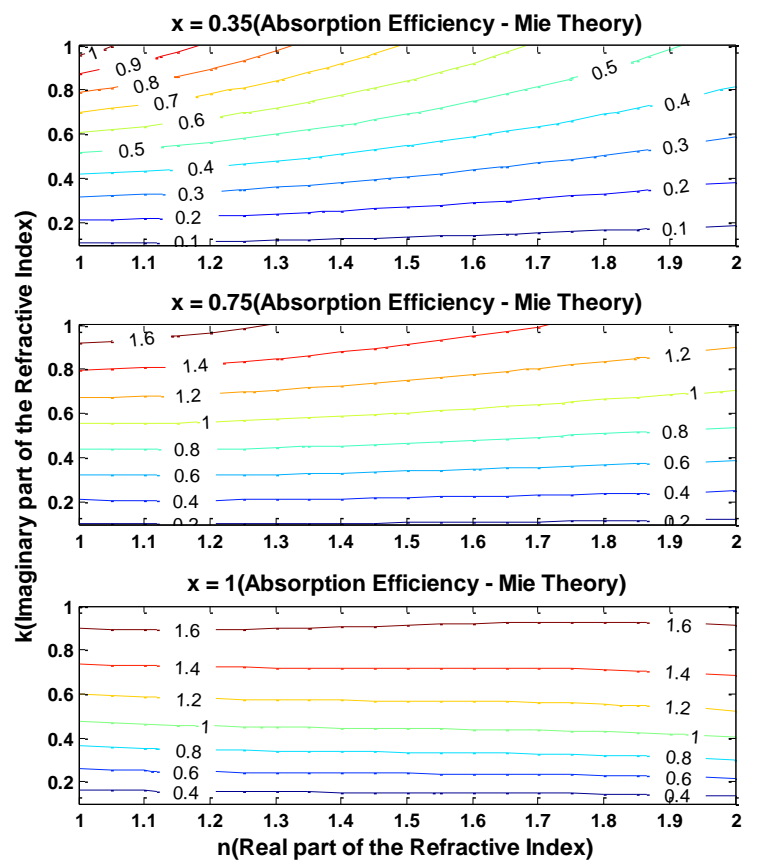

Fig. 2 Effect of refractive index on absorption efficiency when using the Mie theory 

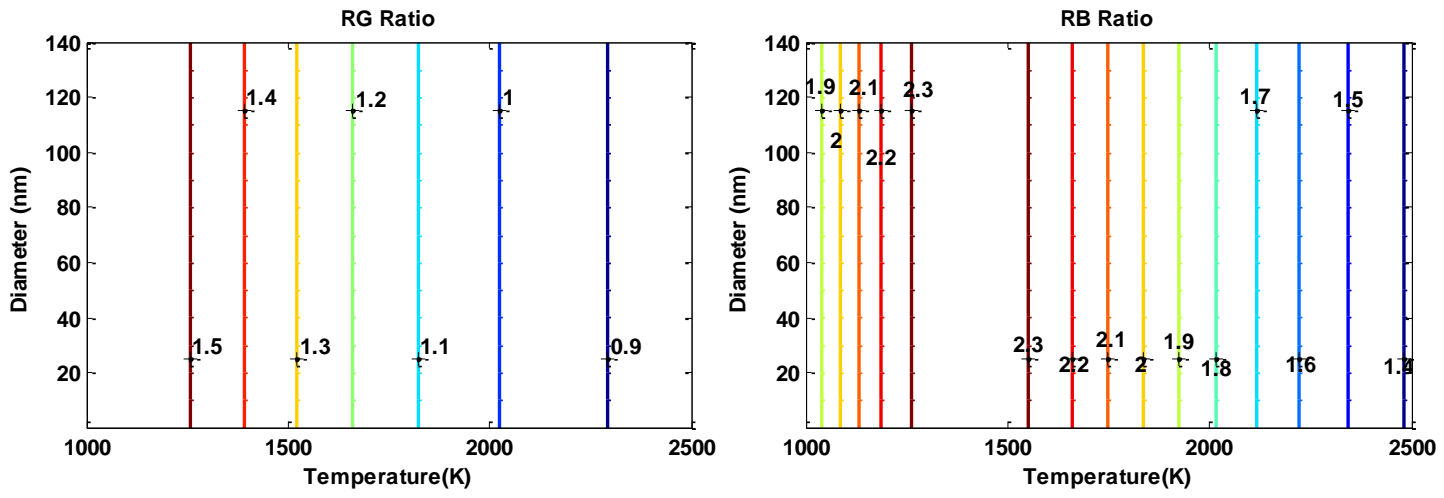

Fig. 3 Plot of the diameter/temperature look-up tables when using the Rayleigh-Gans theory
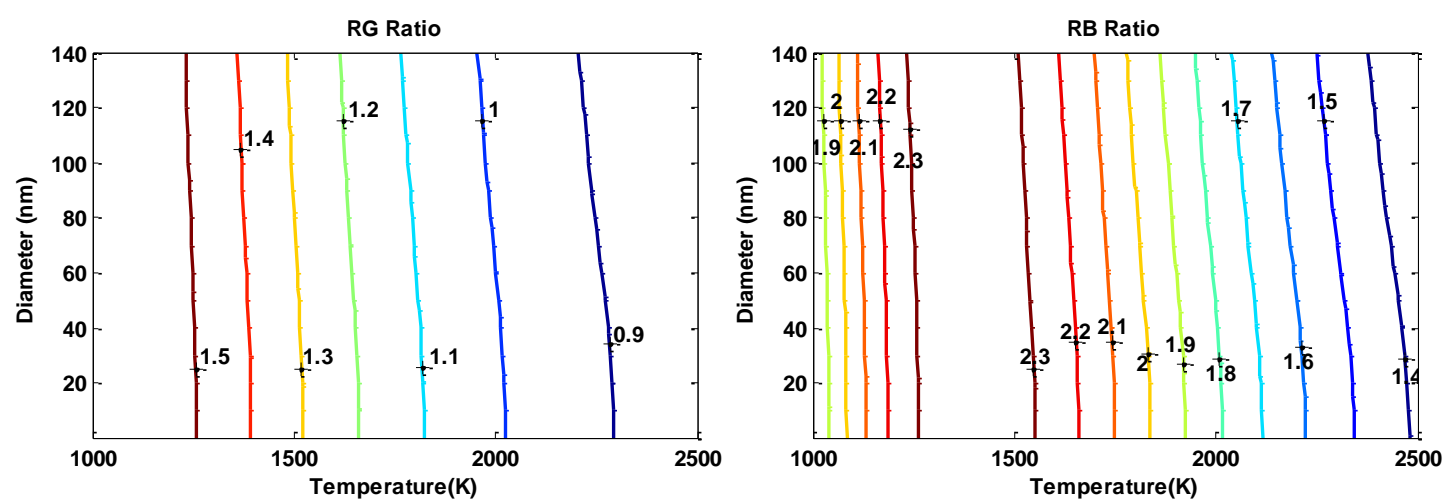

Fig. 4 Plot of the diameter/temperature look-up tables when using the Rayleigh-Gans theory with Penndorf extension correlation
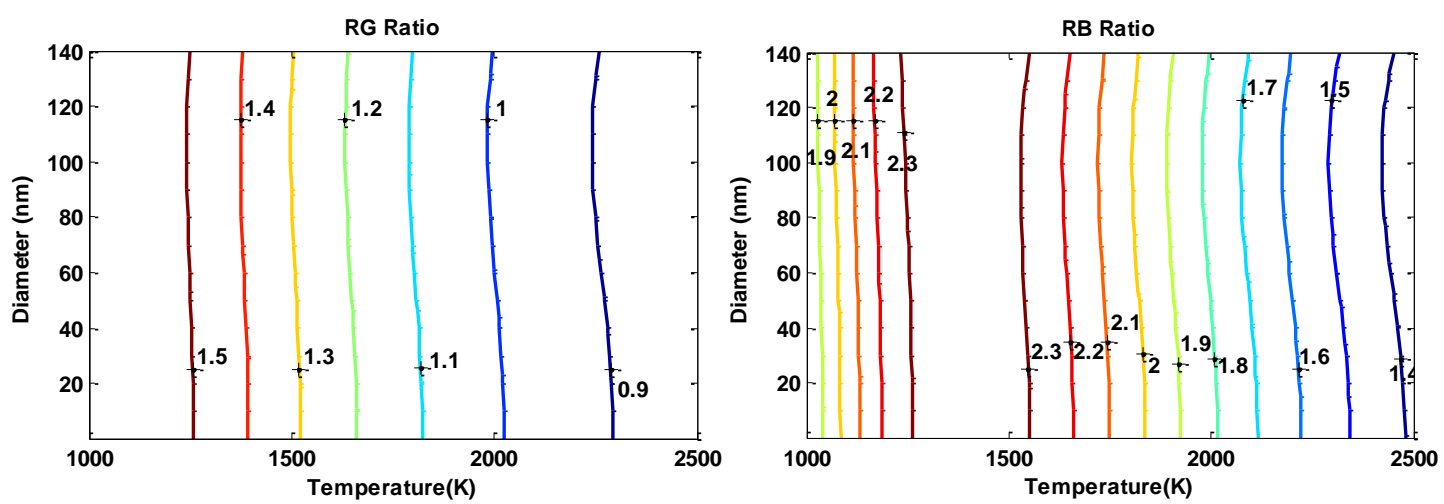

Fig. 5 Plot of the diameter/temperature look-up tables when using the Mie theory 


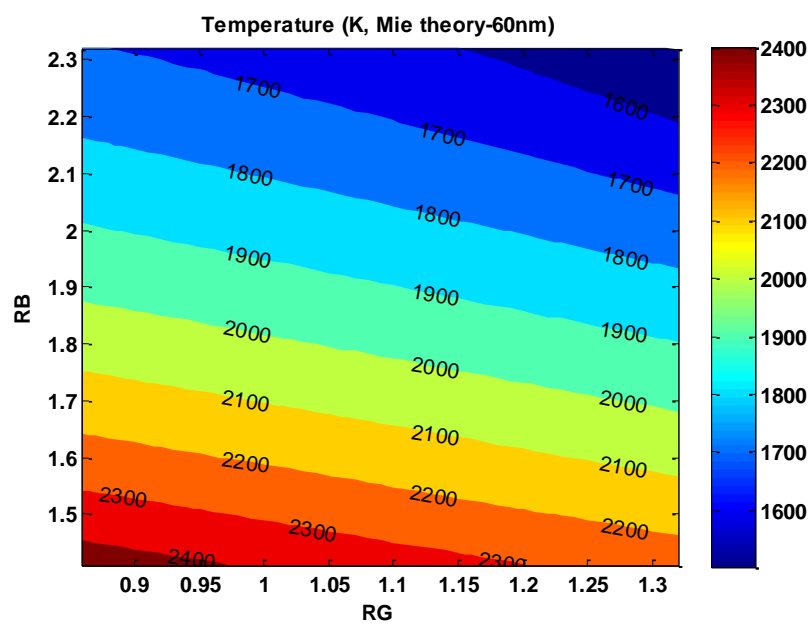

Fig. 6 Contour plot of temperature derived from Mie theory as a function of R/B and R/G colour ratios using a $60 \mathrm{~nm}$ spherical particle diameter.

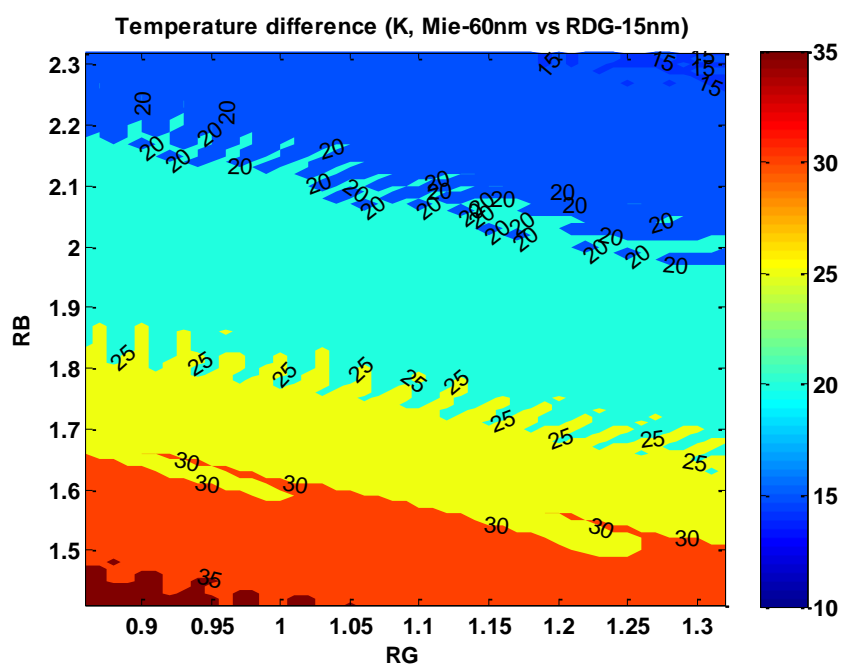

Fig. 7 Temperature difference between Mie theory (60 $\mathrm{nm}$ spherical particle diameter) and RG/RDG-FA theory (15 nm primary particle diameter)

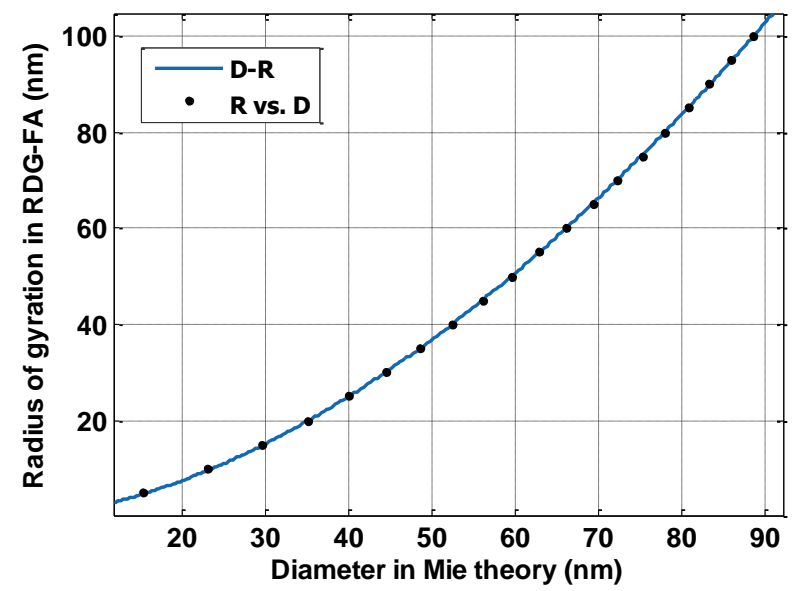

Fig. 8 The correlation between 'diameter' $(D)$ calculated by Mie theory and 'Radius of gyration' $(R)$ in RDG-FA. The solid line is the exponential fitting curve: $R=0.04 D^{1.744}$ 


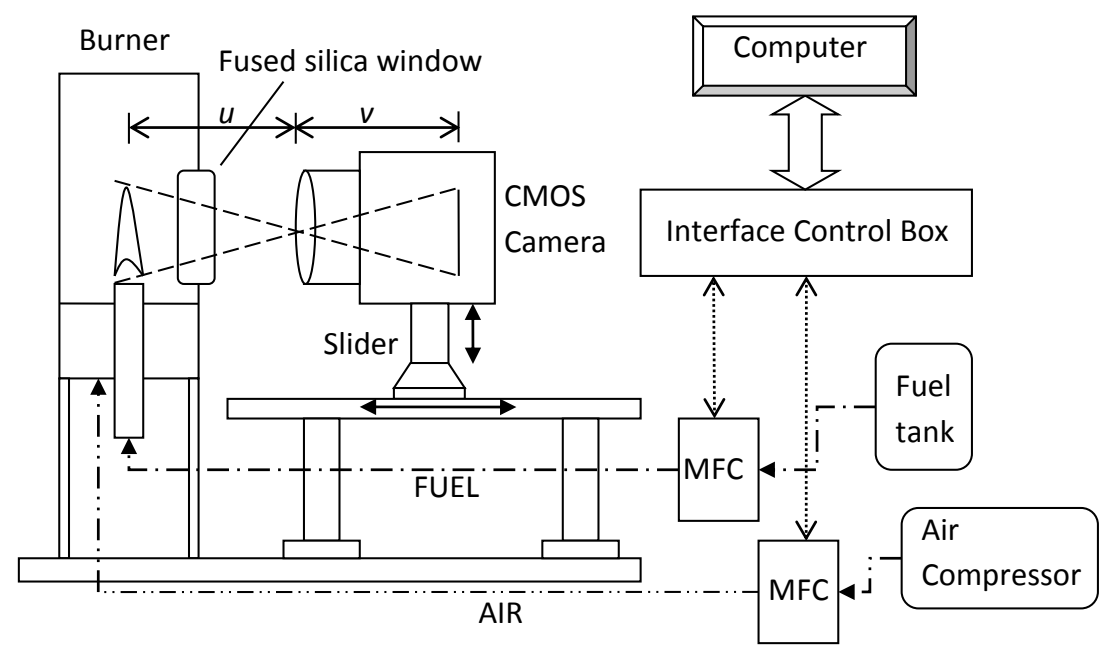

Fig. 9 Schematic of the experimental setup. MFC stands for Mass Flow Controller

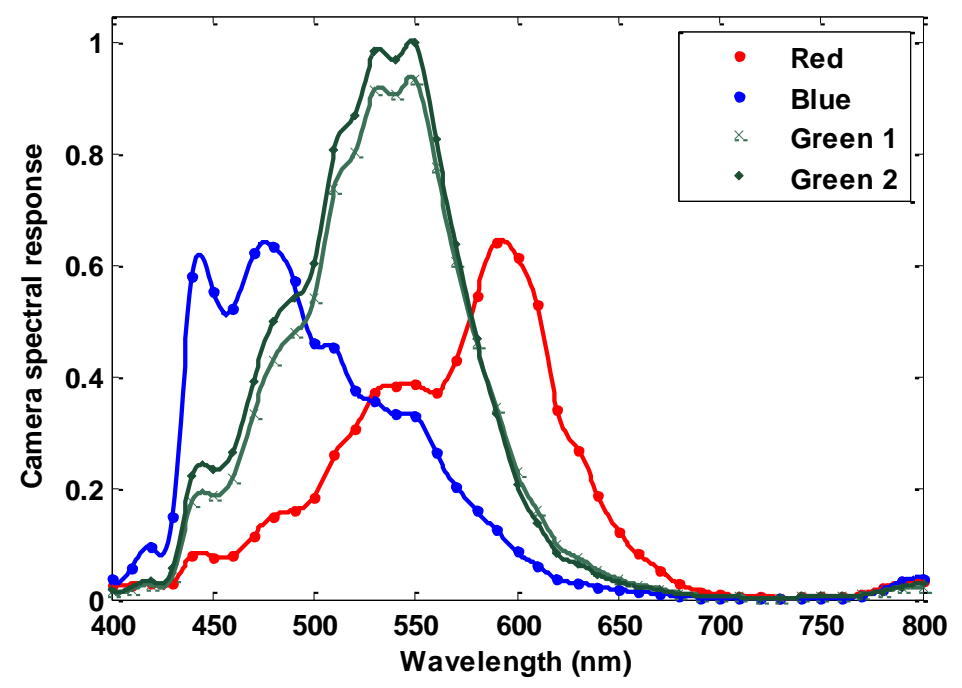

Fig. 10 Camera sensor response curve (the two different green pixels responses are found in the raw image, and this can be attributed to misalignment of the colour filter array and the CMOS sensor). 


\section{Step 1:}

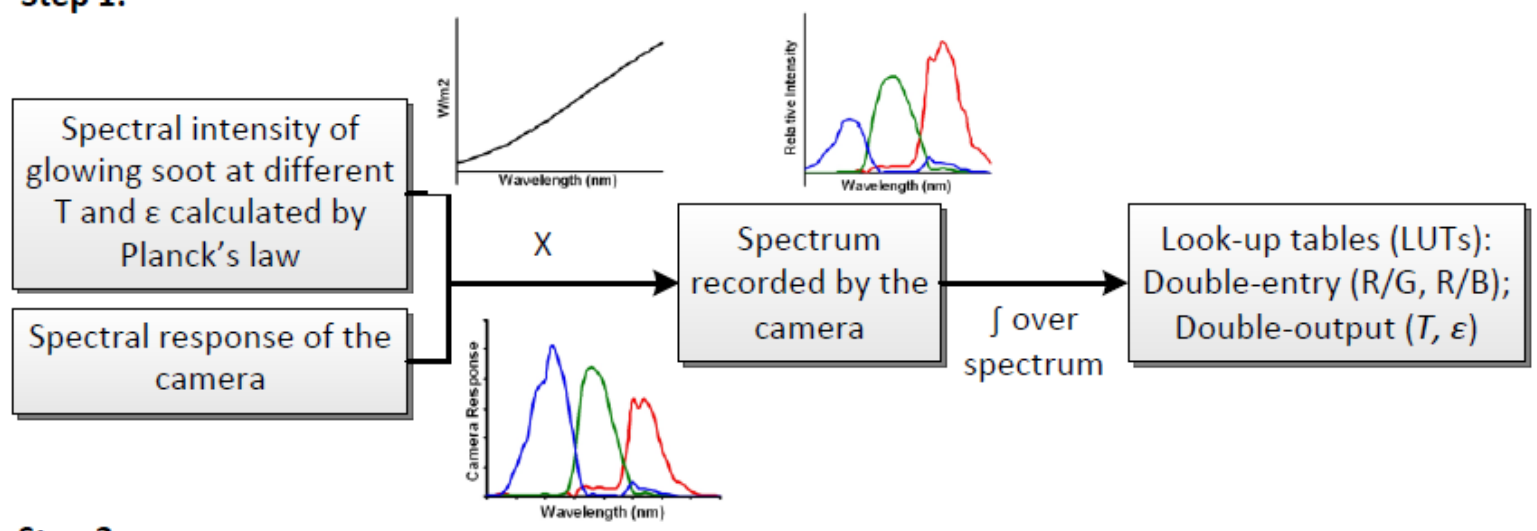

Step 2:

$$
\begin{gathered}
\text { 2D image } \\
(R, G, B)
\end{gathered}
$$

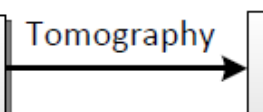

3D colour map $(R, G, B)$
LUTS

3D $T \& \varepsilon$ distribution

Step 3:

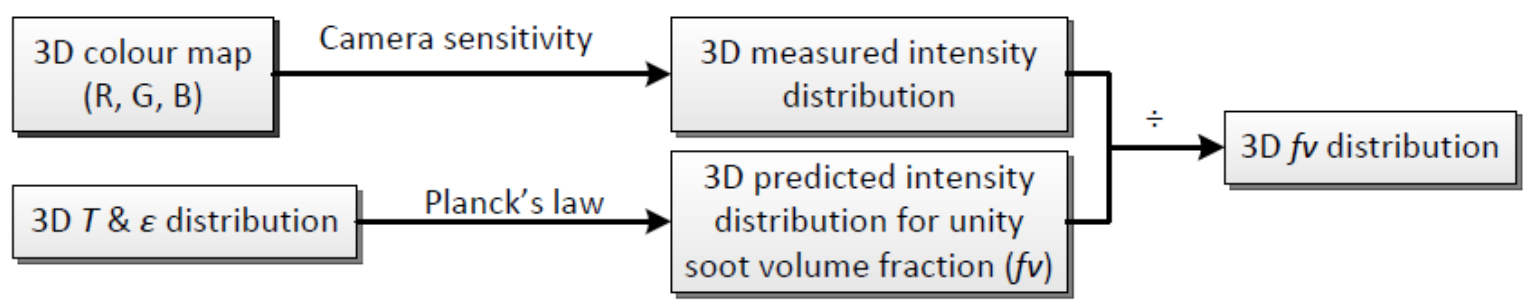

Fig. 11 The signal processing strategy used in the modified three colour pyrometry method 

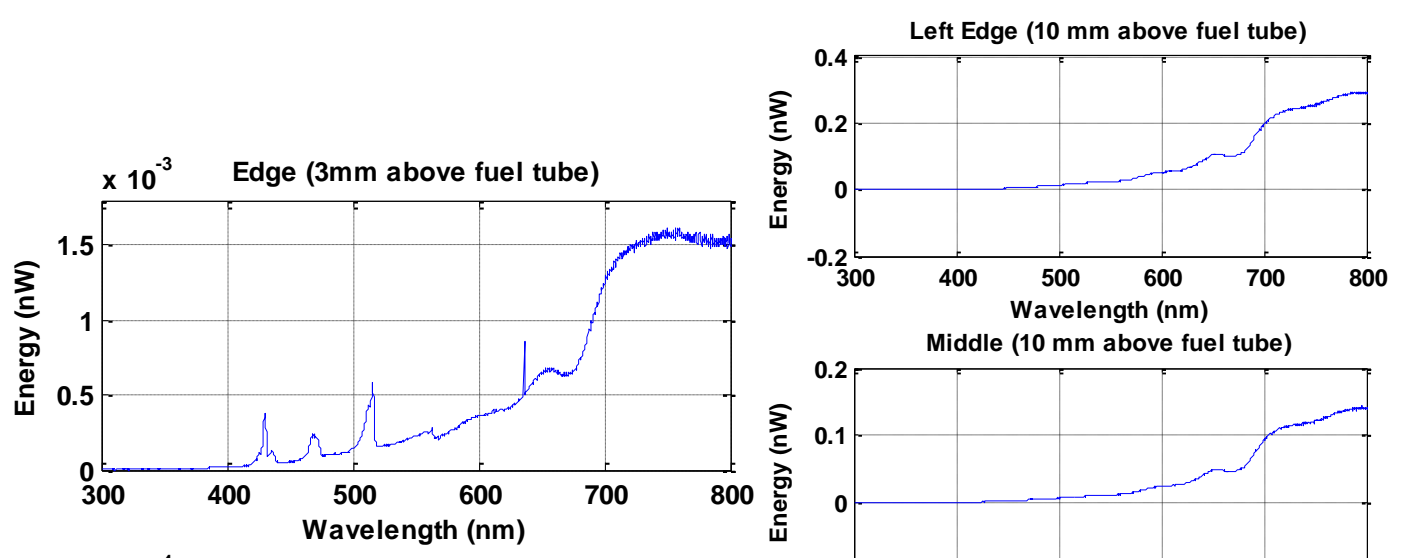

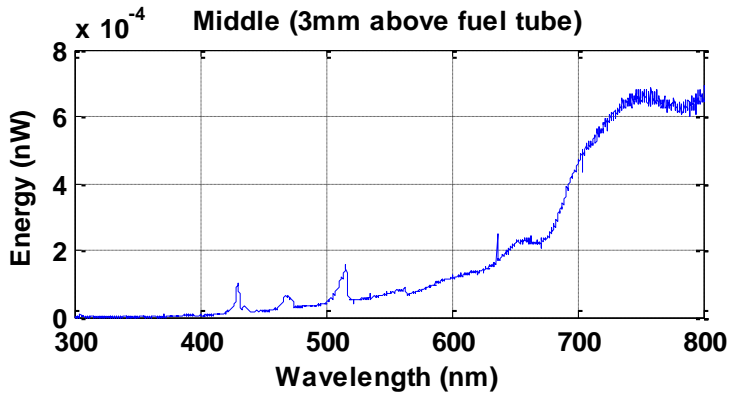

(a)

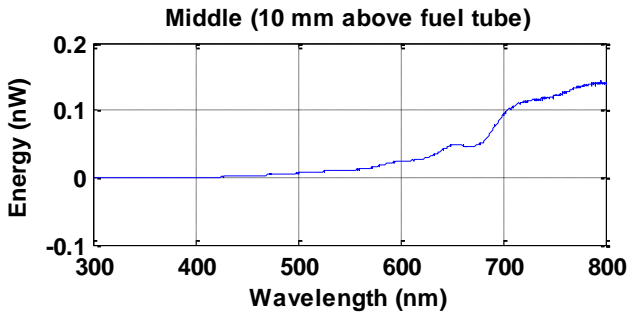

Right Edge (10 $\mathrm{mm}$ above fuel tube)

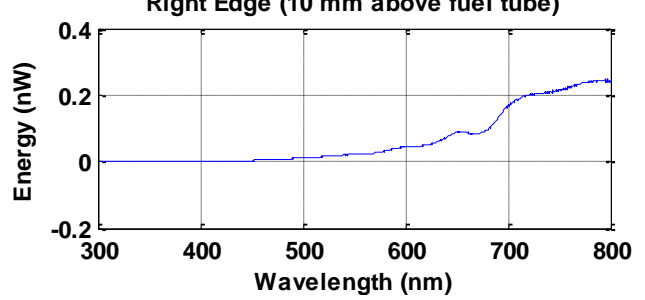

(b)

Fig. 12 Radiation from different parts of an ethylene/air laminar diffusion flame detected by a spectrometer (Fig 10a: $3 \mathrm{~mm}$ above the fuel tube; Fig 10b: $10 \mathrm{~mm}$ above the fuel tube) Note that the colour camera can only record light between 400-700 $\mathrm{nm}$. 

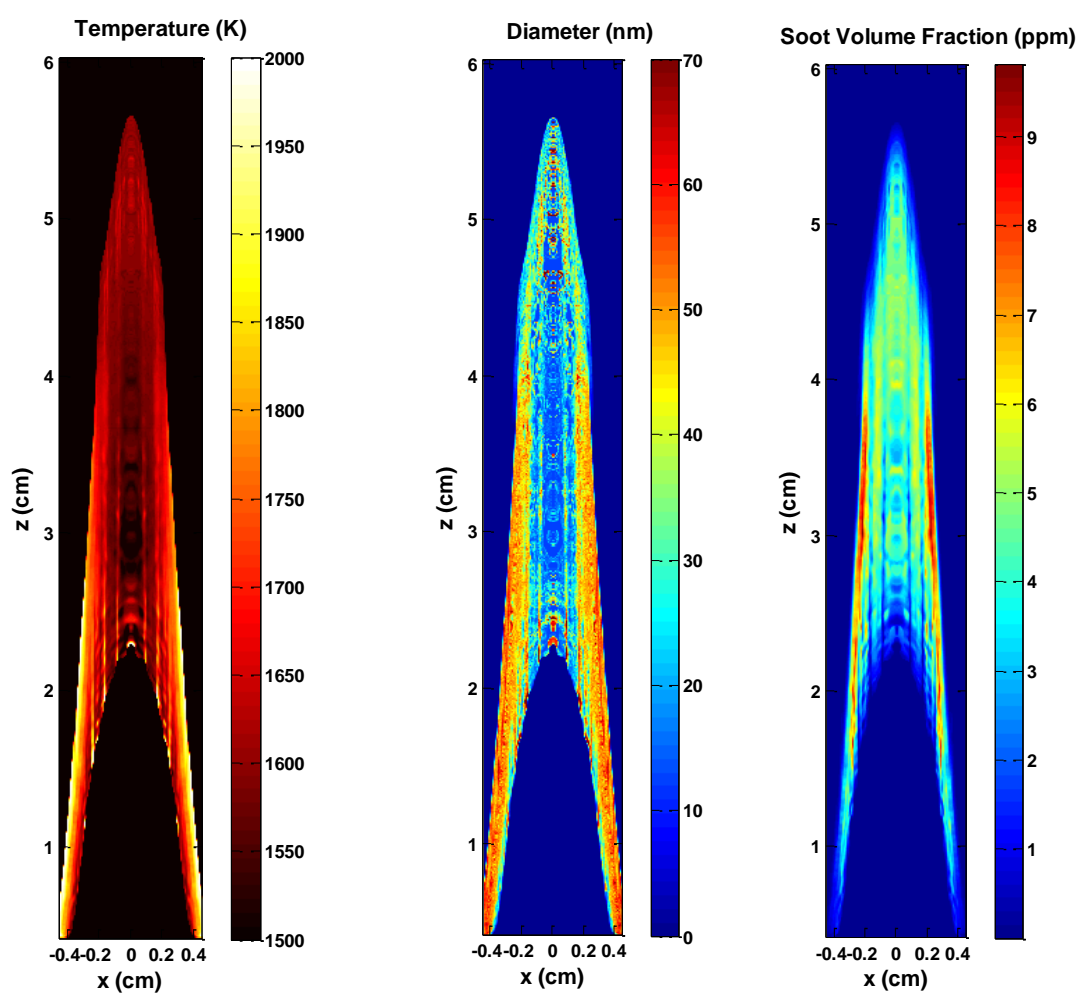

Fig. 13 Temperature, soot diameter and soot volume fraction distributions at the central axis plane calculated by using the Mie theory

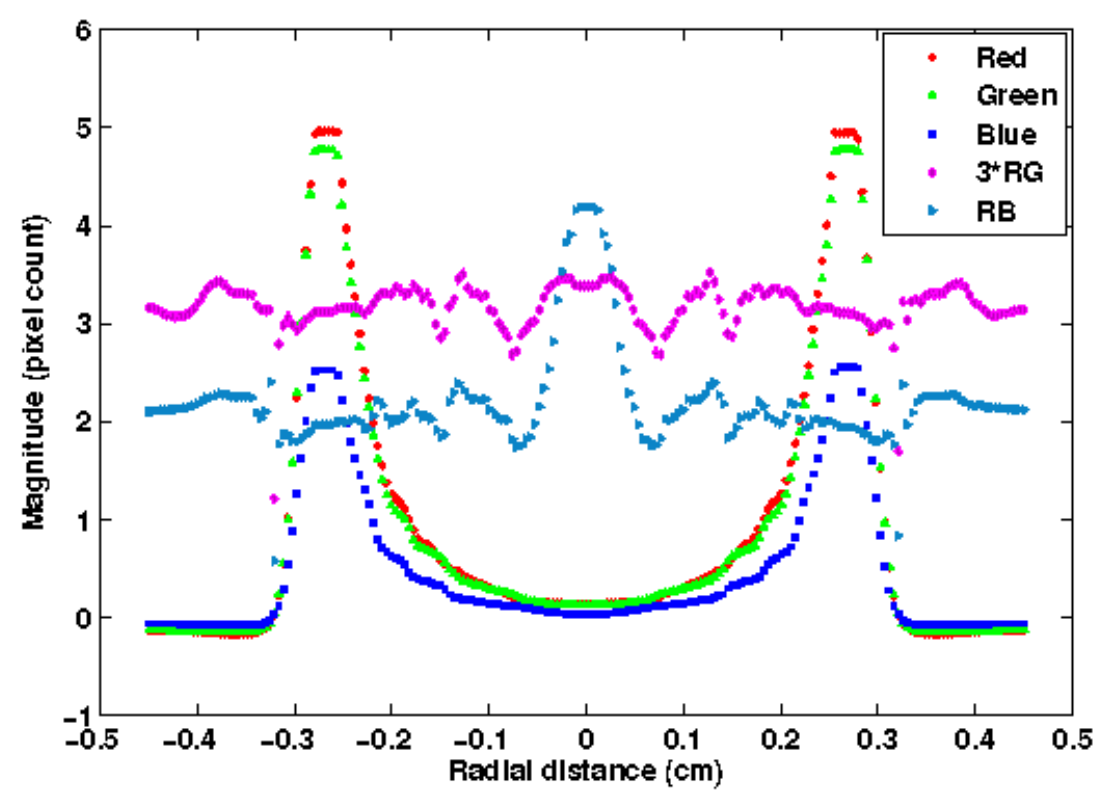

Fig. 14 Typical reconstructed radial colour and colour ratio distributions (in camera pixel count). (3*RG: tripled Red-to-Green ratio; RB: Red-to-Blue ratio) 


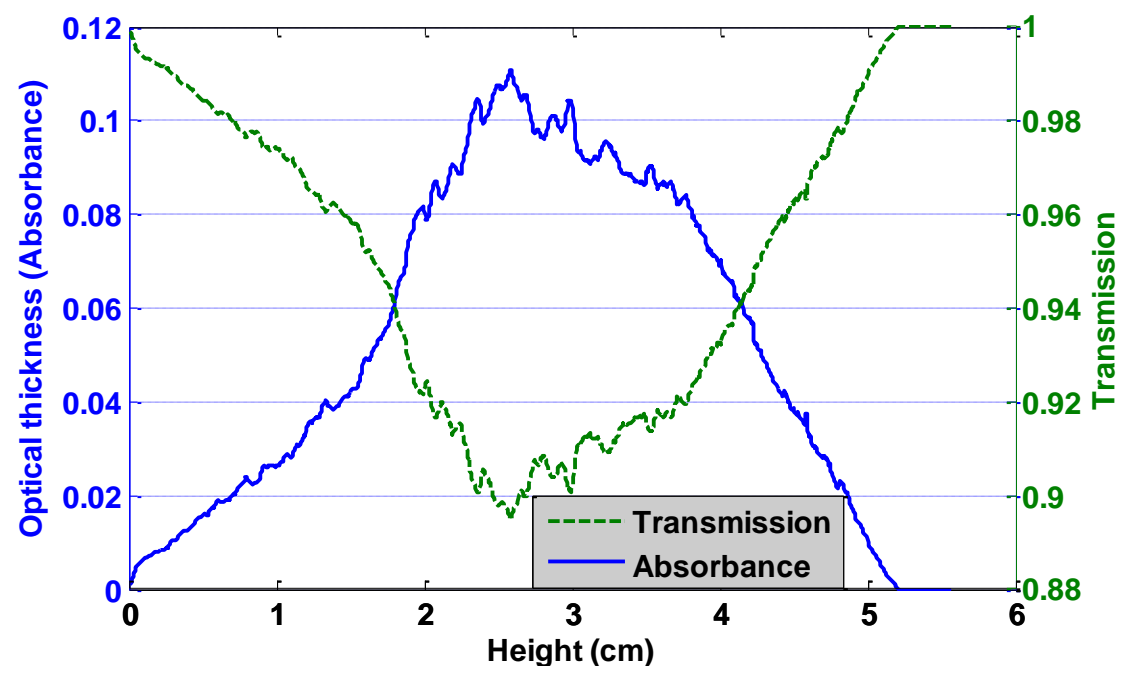

Fig. 15 Optical thickness and transmission at different height 


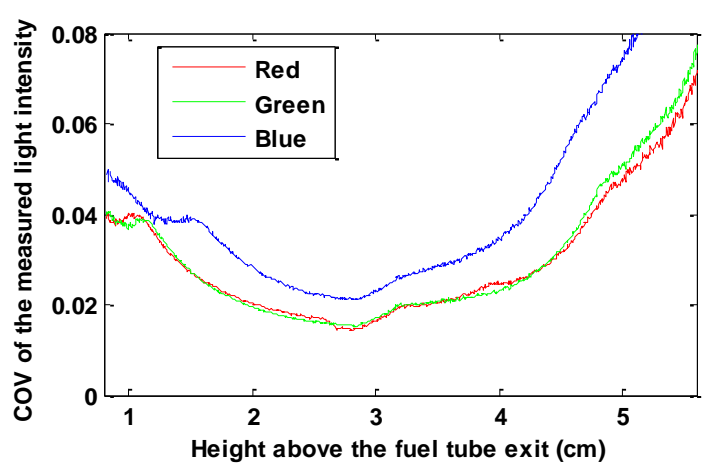

(a)

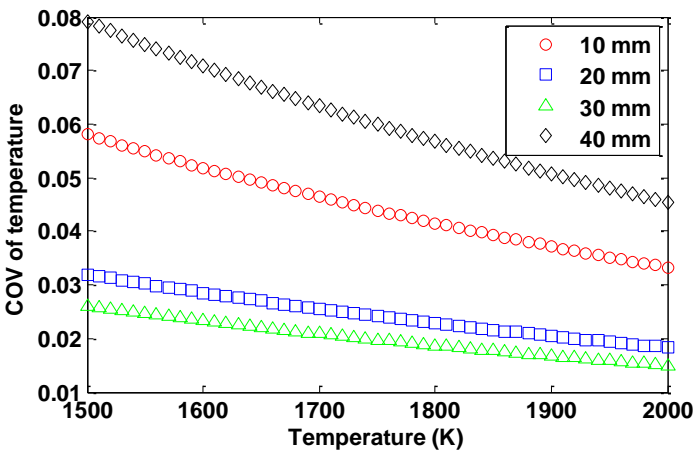

(b)

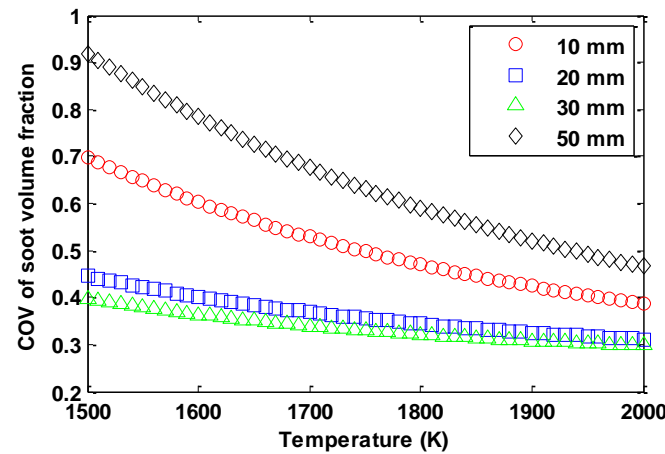

(c)

Fig. 16 (a) COV of the measured light intensity formed from a sequence of 100 images, (b) uncertainty in the calculated temperature and (c) uncertainty in the calculated soot volume fraction. Four data series represent different heights in the flame. 

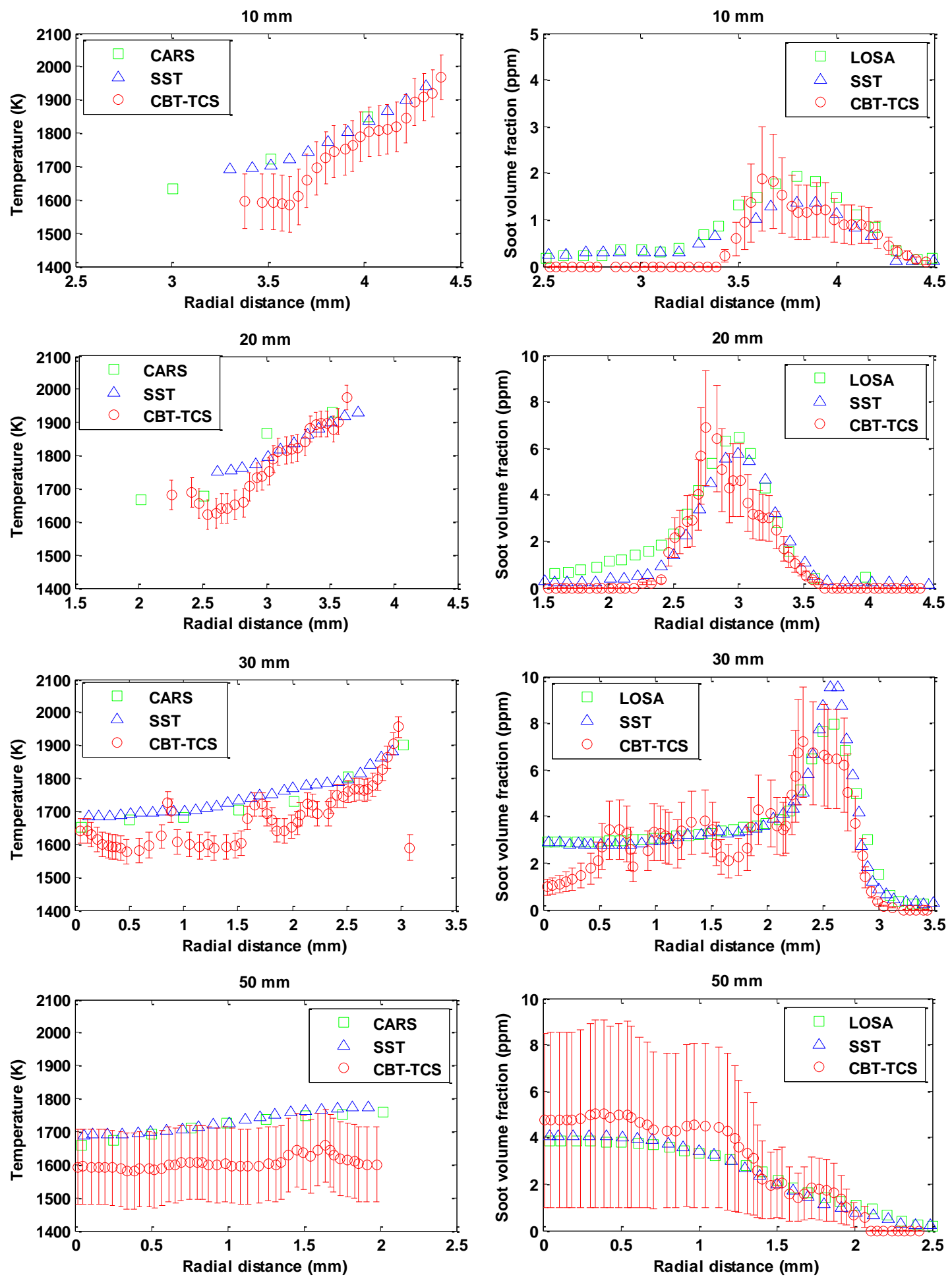

Fig. 17 Comparison of the temperature and soot volume fraction distributions against those measured by Snelling et al. [5] using LOSA (line-of-sight attenuation); SST (2D tomographic soot pyrometry); CARS (Coherent Anti-Stokes Raman Spectroscopy). CBT-TCS: Cone-Beam Tomographic Three Colour Spectrometry is the data reported here. 


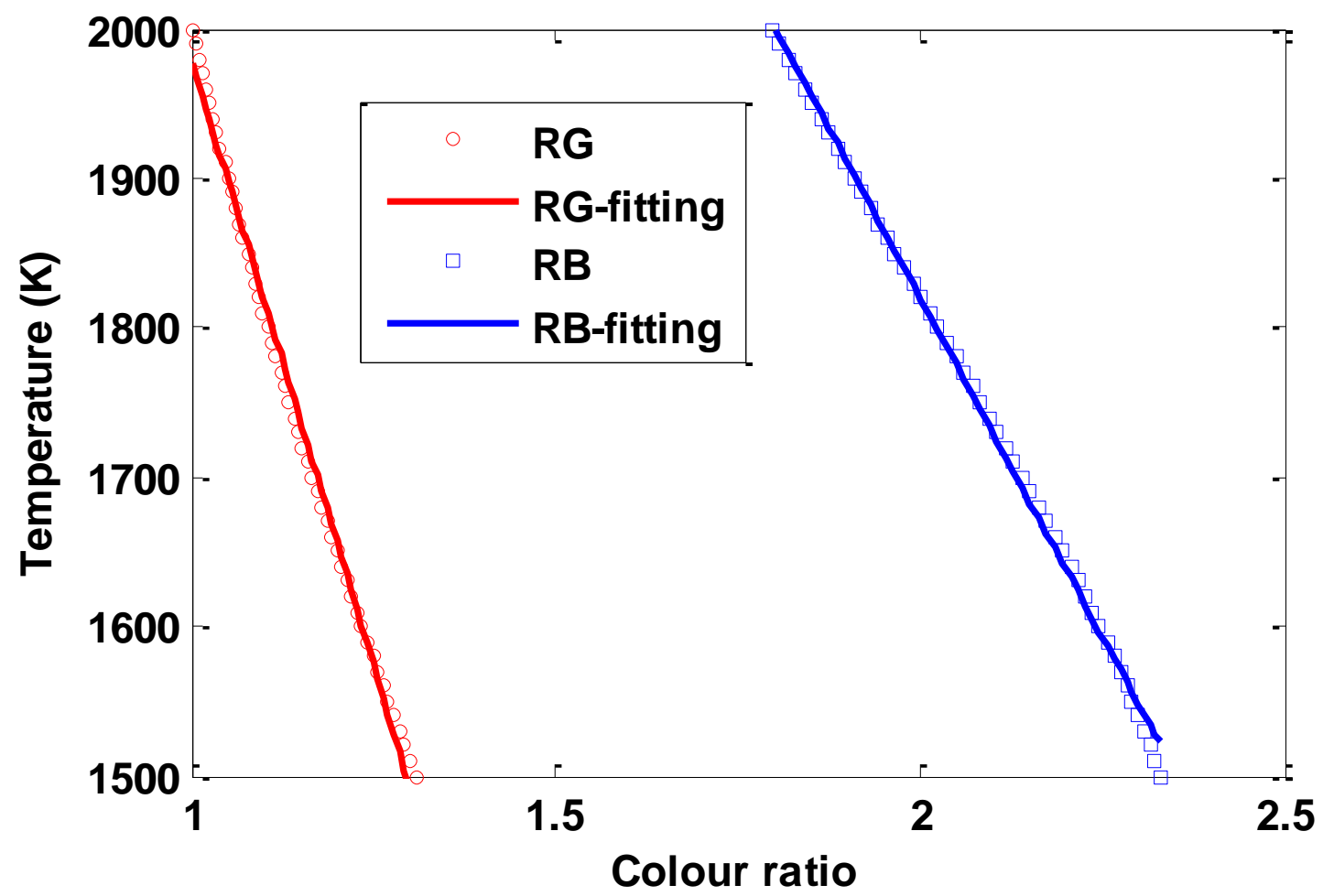

Fig. A1 Temperature as a function of colour ratio. Look up table values are the plotted points, and the line is a linear best fit, which can be seen to closely mimic the trend. (A soot diameter of $50 \mathrm{~nm}$ is assumed here.) 